\title{
Rough singular integrals associated to polynomial curves
}

Yulin Zhang ${ }^{1}$ and Feng Liu' ${ }^{1 *}$

\section{"Correspondence:}

liufeng860314@163.com

'College of Mathematics and

System Sciences, Shandong

University of Science and

Technology, Qingdao, 266590, P.R.

China

\begin{abstract}
In this paper, the authors establish the boundedness of singular integral operators associated to polynomial curves as well as the related maximal operators with rough kernels $\Omega \in H^{1}\left(\mathrm{~S}^{n-1}\right)$ and $h \in \Delta_{\gamma}\left(\mathbb{R}_{+}\right)$for some $\gamma>1$ on the Triebel-Lizorkin spaces. It should be pointed out that the bounds are independent of the coefficients of the polynomials in the definition of the operators. The main results of this paper not only improve and generalize essentially some known results but also complement some recent boundedness results.
\end{abstract}

MSC: Primary 42B20; secondary 42B99

Keywords: Singular integral; Maximal singular integral; Polynomial curves; $H^{1}\left(\mathbf{S}^{n-1}\right)$

\section{Introduction}

It is well known that the Triebel-Lizorkin spaces contain many important function spaces such as Lebesgue spaces, Hardy spaces, Sobolev spaces, and Lipschitz spaces. Over the last several years, a considerable amount of attention has been given to investigate the boundedness for singular integral operators with various rough kernels on the TriebelLizorkin spaces. Particularly, many scholars devoted to studying the bounds for singular integral operators with singularity along various sets under the rough kernels $\Omega \in H^{1}\left(\mathrm{~S}^{n-1}\right)$ and $h \in \Delta_{\gamma}\left(\mathbb{R}_{+}\right)$for some $\gamma>1$. For example, see [10] for the polynomial mappings, [29] for the homogeneous mappings, [27] for the surfaces to revolution. It is unknown whether the singular integral operators associated to polynomial curves under the rough kernels are bounded on the Triebel-Lizorkin spaces. The main purpose of this paper is to address the question. In addition, we establish the bounds for the related maximal singular integral operators on the Lebesgue and Triebel-Lizorkin spaces.

Before stating our main results, let us recall some pertinent definitions, notations, and backgrounds. Let $n \geq 2$ be an integer and let $S^{n-1}$ denote the unit sphere in $\mathbb{R}^{n}$ equipped with the normalized Lebesgue measure $d \sigma$. Let $\Omega \in L^{1}\left(\mathrm{~S}^{n-1}\right)$ be a homogeneous function of degree zero on $\mathbb{R}^{n}$ and satisfy

$$
\int_{S^{n-1}} \Omega(u) d \sigma(u)=0
$$

(c) The Author(s) 2022. This article is licensed under a Creative Commons Attribution 4.0 International License, which permits use, sharing, adaptation, distribution and reproduction in any medium or format, as long as you give appropriate credit to the original author(s) and the source, provide a link to the Creative Commons licence, and indicate if changes were made. The images or other third party material in this article are included in the article's Creative Commons licence, unless indicated otherwise in a credit line to the material. If material is not included in the article's Creative Commons licence and your intended use is not permitted by statutory regulation or exceeds the permitted use, you will need to obtain permission directly from the copyright holder. To view a copy of this licence, visit http://creativecommons.org/licenses/by/4.0/. 
The singular integral operator $T_{h, \Omega}$ is defined as

$$
T_{h, \Omega} f(x):=\text { p.v. } \int_{\mathbb{R}^{n}} \frac{\Omega(y /|y|) h(|y|)}{|y|^{n}} f(x-y) d y,
$$

where $f \in \mathcal{S}\left(\mathbb{R}^{n}\right)$ (the Schwartz class) and $h \in \Delta_{1}\left(\mathbb{R}_{+}\right)$. For $\gamma>0$, the notation $\Delta_{\gamma}\left(\mathbb{R}_{+}\right)$ denotes the set of all measurable functions $h$ on $\mathbb{R}_{+}:=(0, \infty)$ satisfying

$$
\|h\|_{\Delta_{\gamma}\left(\mathbb{R}_{+}\right)}=\sup _{R>0}\left(\frac{1}{R} \int_{0}^{R}|h(t)|^{\gamma} d t\right)^{1 / \gamma}<\infty .
$$

It is not difficult to see that $L^{\infty}\left(\mathbb{R}_{+}\right)=\Delta_{\infty}\left(\mathbb{R}_{+}\right) \subsetneq \Delta_{\gamma_{2}}\left(\mathbb{R}_{+}\right) \subsetneq \Delta_{\gamma_{1}}\left(\mathbb{R}_{+}\right)$for $0<\gamma_{1}<\gamma_{2}<\infty$. For the sake of simplicity, we denote $T_{h, \Omega}=T_{\Omega}$ when $h \equiv 1$.

The theory of singular integral originated in Calderón and Zygmund's work [4] in which they used the rotation method to establish the $L^{p}\left(\mathbb{R}^{n}\right)(1<p<\infty)$ of $T_{\Omega}$ if $\Omega \in L \log L\left(\mathrm{~S}^{n-1}\right)$. Since then, more and more scholars have been devoted to studying the boundedness of singular integrals with various rough kernels. Particularly, Coifman and Weiss [12] proved that $T_{\Omega}$ is of type $(p, p)$ for $1<p<\infty$ if $\Omega \in H^{1}\left(\mathrm{~S}^{n-1}\right)$ (see also [15]). It was remarkable that $\Omega \in H^{1}\left(\mathrm{~S}^{n-1}\right)$ turned out to be the weakest size condition for the $L^{p}$ boundedness of $T_{\Omega}$ up to now. Later on, an active extension to the theory was due to Fefferman [23] who discovered that the Calderón-Zygmund rotation method is no longer available if $T_{h, \Omega}$ is also rough in the radial direction, for instance $h \in L^{\infty}\left(\mathbb{R}_{+}\right)$, so that new methods must be addressed. More precisely, Fefferman [23] showed that $T_{h, \Omega}$ is of type $(p, p)$ for $1<p<\infty$ if $\Omega \in \operatorname{Lip}_{\alpha}\left(S^{n-1}\right)$ for some $\alpha>0$ and $h \in L^{\infty}\left(\mathbb{R}_{+}\right)$. Fefferman's result was later improved by Namazi [32] by assuming $\Omega \in L^{q}\left(\mathrm{~S}^{n-1}\right)$ for some $q>1$ instead of $\Omega \in \operatorname{Lip}_{\alpha}\left(\mathrm{S}^{n-1}\right)$. Meanwhile, Duoandikoetxea and Rubio de Francia [16] used the Littlewood-Paley theory to improve the results to the case $\Omega \in L^{q}\left(\mathrm{~S}^{n-1}\right)$ for any $q>1$ and $h \in \Delta_{2}\left(\mathbb{R}_{+}\right)$. The boundedness for rough singular integral operators on Tribel-Lizorkin spaces has also been studied extensively by many authors. In 2002, Chen, Fan, and Ying [5] first showed that $T_{\Omega}$ is bounded on $\dot{F}_{\alpha}^{p, q}\left(\mathbb{R}^{n}\right)$ if $\Omega \in L^{r}\left(\mathrm{~S}^{n-1}\right)$ for some $r>1$. Later on, the result was extended and improved by many authors. For example, see $[2,6]$ for the case $\Omega \in \mathcal{F}_{\beta}\left(\mathrm{S}^{n-1}\right)$ (the Grafakos-Stefanov function class in $[25])$, $[9,10]$ for the case $\Omega \in H^{1}\left(S^{n-1}\right)$.

For the operators $T_{\Omega}$ and $T_{h, \Omega}$, the singularities are along the diagonal $\{x=y\}$. However, many problems in analysis have led one to consider singular integral operators with singularity along more general sets. One of the principal motivations for the study of such operators is the requirements of several complex variables and large classes of "subelliptic" equations (see $[37,39]$ ). So more and more scholars are devoted to studying the $L^{p}$ bounds for rough singular integral operators with singularity along various sets. For example, see $[3,22,34]$ for polynomial mappings, $[17,19]$ for real-analytic submanifolds, $[11,28]$ for homogeneous mappings, $[1,18,20,26]$ for polynomial curves. Other interesting works can be found in $[7,8,35,36,42]$, among others.

In this paper we focus on the singular integrals associated to polynomial curves with rough kernels. Let $h, \Omega$ be given as in (1.2) and $P$ be a real polynomial on $\mathbb{R}$ satisfying $P(0)=0$. For a function $\varphi: \mathbb{R}_{+} \rightarrow \mathbb{R}$, we define the singular integral operator associated to polynomial compound curves $\left\{P(\varphi(|y|)) y /|y| ; y \in \mathbb{R}^{n}\right\}$ by

$$
T_{h, \Omega, P, \varphi} f(x):=\text { p.v. } \int_{\mathbb{R}^{n}} f(x-P(\varphi(|y|)) y /|y|) \frac{\Omega(y /|y|) h(|y|)}{|y|^{n}} d y,
$$


where $f \in \mathcal{S}\left(\mathbb{R}^{n}\right)$. When $\varphi(t) \equiv t$, we denote $T_{h, \Omega, P, \varphi}=T_{h, \Omega, P}$. Particularly, $T_{h, \Omega, P}=T_{h, \Omega}$ when $P(t) \equiv t$. In 1997, Fan and Pan [20] first established the $L^{2}$ boundedness for $T_{h, \Omega, P}$ if $h \in L^{\infty}\left(\mathbb{R}_{+}\right)$and $\Omega \in H^{1}\left(\mathrm{~S}^{n-1}\right)$. Subsequently, Al-Hasan and Pan [1] improved the result by establishing the following.

Theorem $\mathbf{A}$ ([1]) Let $h \in L^{\infty}\left(\mathbb{R}_{+}\right)$and $\Omega \in H^{1}\left(\mathrm{~S}^{n-1}\right)$ satisfy (1.1). Then, for $1<p<\infty$, there exists a constant $C>0$ independent of $h, \Omega$ and the coefficients of $P$ such that

$$
\left\|T_{h, \Omega, P} f\right\|_{L^{p}\left(\mathbb{R}^{n}\right)} \leq C\|h\|_{L^{\infty}\left(\mathbb{R}_{+}\right)}\|\Omega\|_{H^{1}\left(S^{n-1}\right)}\|f\|_{L^{p}\left(\mathbb{R}^{n}\right)}, \quad \forall f \in L^{p}\left(\mathbb{R}^{n}\right) .
$$

Later on, the $L^{p}$ mapping properties for $T_{h, \Omega, P}$ have been investigated by many authors. For example, see [18] for the case $h \equiv 1$ and $\Omega \in \mathcal{F}_{\beta}\left(\mathrm{S}^{n-1}\right)$, [26] for the case $\Omega \in L \log L\left(\mathrm{~S}^{n-1}\right)$.

Based on (2.4) and Theorem A, a natural question is the following.

Question 1.1 Is $T_{h, \Omega, P}$ bounded on $F_{\alpha}^{p, q}\left(\mathbb{R}^{n}\right)$ if $h \in \Delta_{\gamma}\left(\mathbb{R}_{+}\right)$for some $\gamma \in(1, \infty]$ and $\Omega \in$ $H^{1}\left(\mathrm{~S}^{n-1}\right)$ ?

Our investigation will not only address this question, but also deal with a more general class of operators. More specifically, we have the following result.

Theorem 1.1 Let $P$ be a real polynomial on $\mathbb{R}$ satisfying $P(0)=0$ and $\varphi \in \mathfrak{F}_{1}$ or $\mathfrak{F}_{2}$. Here, $\mathfrak{F}_{1}$ (resp., $\left.\mathfrak{F}_{2}\right)$ is the set of all functions $\phi: \mathbb{R}_{+} \rightarrow \mathbb{R}$ satisfying the following condition (a) (resp., (b)):

(a) $\phi$ is an increasing $\mathcal{C}^{1}$ function such that $t \phi^{\prime}(t) \geq C_{\phi} \phi(t)$ and $\phi(2 t) \leq c_{\phi} \phi(t)$ for all $t>0$, where $C_{\phi}$ and $c_{\phi}$ are independent of $t$.

(b) $\phi$ is a decreasing $\mathcal{C}^{1}$ function such that $t \phi^{\prime}(t) \leq-C_{\phi} \phi(t)$ and $\phi(t) \leq c_{\phi} \phi(2 t)$ for all $t>0$, where $C_{\phi}$ and $c_{\phi}$ are independent of $t$.

Suppose that $\Omega \in H^{1}\left(\mathrm{~S}^{n-1}\right)$ satisfies (1.1) and $h \in \Delta_{\gamma}\left(\mathbb{R}_{+}\right)$for some $\gamma \in(1, \infty]$. Then

(i) For $\alpha \in \mathbb{R}$ and $(1 / p, 1 / q) \in \mathcal{R}_{\gamma}$, there exists a constant $C>0$ independent of $h, \gamma, \Omega$ and the coefficients of $P$ such that

$$
\left\|T_{h, \Omega, P, \varphi} f\right\|_{\dot{F}_{\alpha}^{p, q}\left(\mathbb{R}^{n}\right)} \leq C \gamma^{\prime}\|h\|_{\Delta_{\gamma}\left(\mathbb{R}_{+}\right)}\|\Omega\|_{H^{1}\left(S^{n-1}\right)}\|f\|_{\dot{F}_{\alpha}^{p, q}\left(\mathbb{R}^{n}\right)}
$$

Here, $\mathcal{R}_{\gamma}$ is the interior of the convex hull of three squares $\left(\frac{1}{2}, \frac{1}{2}+\frac{1}{\max \left\{2, \gamma^{\prime}\right\}}\right)^{2}$, $\left(\frac{1}{2}-\frac{1}{\max \left\{2, \gamma^{\prime}\right\}}, \frac{1}{2}\right)^{2}$, and $\left(\frac{1}{2 \gamma}, 1-\frac{1}{2 \gamma}\right)^{2}$.

(ii) For $\alpha>0$ and $(1 / p, 1 / q) \in \mathcal{R}_{\gamma}$, there exists a constant $C>0$ independent of $h, \gamma, \Omega$ and the coefficients of $P$ such that

$$
\left\|T_{h, \Omega, P, \varphi} f\right\|_{F_{\alpha}^{p, q}\left(\mathbb{R}^{n}\right)} \leq C \gamma^{\prime}\|h\|_{\Delta_{\gamma}\left(\mathbb{R}_{+}\right)}\|\Omega\|_{H^{1}\left(\mathrm{~S}^{n-1}\right)}\|f\|_{F_{\alpha}^{p, q}\left(\mathbb{R}^{n}\right)}
$$

Remark 1.1 There are some model examples in the class $\mathfrak{F}_{1}$ such as $t^{\alpha}(\alpha>0), t^{\alpha}(\ln (1+$ $t))^{\beta}(\alpha, \beta>0), t \ln \ln (e+t)$, real-valued polynomials $P$ on $\mathbb{R}$ with positive coefficients and $P(0)=0$, and so on. We now give examples in the class $\mathfrak{F}_{2}$ such as $t^{\delta}(\delta<0)$ and $t^{-1} \ln (1+$ $1 / t)$. It was pointed out in [26] that for $\varphi \in \mathfrak{F}_{1}$ (or $\mathfrak{F}_{2}$ ) there exists a constant $B_{\varphi}>1$ such that $\varphi(2 t) \geq B_{\varphi} \varphi(t)\left(\right.$ or $\left.\varphi(t) \geq B_{\varphi} \varphi(2 t)\right)$. 
Remark 1.2 (i) It is clear that $\mathcal{R}_{\gamma_{1}} \subsetneq \mathcal{R}_{\gamma_{2}}$ for $\gamma_{1}<\gamma_{2}$ and $\mathcal{R}_{\infty}=(0,1) \times(0,1)$. In view of (2.4), we see that Theorem 1.1 essentially improved and generalized Theorem A.

(ii) Our methods used to deal with Fourier transform estimates of some measures are different from those in the proof of Theorem A. In fact, the authors in [1] used the $T T^{*}$ method to prove Theorem A. However, the $T T^{*}$ method is not needed in the proof of Theorem 1.1.

(iii) Part (i) of Theorem 1.1 improved and generalized Theorem 1 in [9], in which the authors showed that $T_{h, \Omega}$ is bounded on $\dot{F}_{\alpha}^{p, q}\left(\mathbb{R}^{n}\right)$ for $\alpha \in \mathbb{R}$ and $1<p, q<\infty$, provided that $h \in L^{\infty}\left(\mathbb{R}_{+}\right)$and $\Omega \in H^{1}\left(S^{n-1}\right)$.

(iv) Theorem 1.1 is new, even in the special case $h \equiv 1$ or $\alpha=0, q=2, \varphi(t) \equiv t$, or $P(t) \equiv t$.

The second motivation of this paper is concerned with the $L^{p}$ boundedness of maximal truncated singular integrals associated to polynomial curves. Let $h, \Omega, P, \varphi$ be given as in (1.3). The maximal truncated singular integral operator $T_{h, \Omega, P, \varphi}^{*}$ is defined by

$$
T_{h, \Omega, P, \varphi}^{*} f(x):=\sup _{\epsilon>0}\left|\int_{|y|>\epsilon} f(x-P(\varphi(|y|)) y /|y|) \frac{\Omega(y /|y|) h(|y|)}{|y|^{n}} d y\right|,
$$

where $f \in \mathcal{S}\left(\mathbb{R}^{n}\right)$. The type of operator $T_{h, \Omega, P, \varphi}^{*}$ was first studied by Fan, Guo, and Pan [18] who proved that $T_{h, \Omega, P, \varphi}^{*}$ is bounded on $L^{p}\left(\mathbb{R}^{n}\right)$ for $(2 \beta-1) /(2 \beta-2)<p<2 \beta-1$ if $h \equiv 1$, $\varphi(t) \equiv t$, and $\Omega \in \mathcal{F}_{\beta}\left(\mathrm{S}^{n-1}\right)$ for some $\beta>3 / 2$. Recently, Liu [26] proved that $T_{h, \Omega, P, \varphi}^{*}$ is of type $(p, p)$ for $1<p<\infty$, provided that $\varphi \in \mathfrak{F}_{1}$ or $\mathfrak{F}_{2}, \Omega \in L \log L\left(\mathrm{~S}^{n-1}\right)$ and $h$ satisfies certain radial condition.

Based on (2.1), (2.2) and the results related to $T_{h, \Omega, P, \varphi}^{*}$, a natural question is the following.

Question 1.2 Is $T_{h, \Omega, P, \varphi}^{*}$ bounded on $L^{p}\left(\mathbb{R}^{n}\right)$ for some $p>1$ under the same conditions of Theorem 1.1?

This question can be addressed by the following.

Theorem 1.2 Let $P, \varphi$ be given as in Theorem 1.1. Suppose that $\Omega \in H^{1}\left(\mathrm{~S}^{n-1}\right)$ satisfies (1.1) and $h \in \Delta_{\gamma}\left(\mathbb{R}_{+}\right)$for some $\gamma \in(4 / 3, \infty]$. Then there exists a constant $C>0$ independent of $h, \gamma, \Omega$ and the coefficients of $P$ such that

$$
\left\|T_{h, \Omega, P, \varphi}^{*} f\right\|_{L^{p}\left(\mathbb{R}^{n}\right)} \leq C \gamma^{\prime}\|h\|_{\Delta_{\gamma}\left(\mathbb{R}_{+}\right)}\|\Omega\|_{H^{1}\left(S^{n-1}\right)}\|f\|_{L^{p}\left(\mathbb{R}^{n}\right)}, \quad \forall f \in L^{p}\left(\mathbb{R}^{n}\right) .
$$

Here, $p \in\left(\gamma^{\prime}, \infty\right)$ if $\gamma \geq 2$ or $p \in\left(\gamma^{\prime}, 2 \gamma^{\prime} /\left(\gamma^{\prime}-2\right)\right)$ if $\gamma \in(4 / 3,2)$.

Remark 1.3 Theorem 1.2 is new, even in the special case $h \equiv 1$ or $\varphi(t) \equiv t$. It is unknown whether the operator $T_{h, \Omega, P, \varphi}$ appearing in Theorem 1.2 is bounded on $L^{p}\left(\mathbb{R}^{n}\right)$ for some $p>1$ if $\gamma \in(1,4 / 3]$, even in the special case $\varphi(t)=t$, which is very interesting.

The third motivation of this paper is concerned with the boundedness of maximal truncated singular integrals associated to polynomial curves on Triebel-Lizorkin spaces. The first work related to the boundedness for maximal singular integral operator on Triebel-Lizorkin spaces was due to Zhang and Chen [43], who showed that the maximal singular integral operator is bounded on $\dot{F}_{\alpha}^{p, q}\left(\mathbb{R}^{n}\right)$ and $F_{\alpha}^{p, q}\left(\mathbb{R}^{n}\right)$ for $0<\alpha<1$ and 
$1<p, q<\infty$ by assuming that $\Omega \in H^{1}\left(\mathrm{~S}^{n-1}\right)$. Recently, Liu, Xue, and Yabuta [30] established the boundedness for the maximal singular integral operators associated to polynomial mappings on Triebel-Lizorkin spaces under the conditions $h \in \Delta_{\gamma}\left(\mathbb{R}_{+}\right)$with some $\gamma>1$ and $\Omega \in L \log L\left(\mathrm{~S}^{n-1}\right)$. Very recently, the authors [31] obtained the boundedness for $T_{h, \Omega, P, \varphi}^{*}$ on Triebel-Lizorkin spaces, provided that $h \equiv 1, \Omega \in \mathcal{F}_{\beta}\left(\mathrm{S}^{n-1}\right)$ with some $\beta>3 / 2$ and $\varphi \in \mathfrak{F}_{3}$, where $\mathfrak{F}_{3}$ is the set of all functions $\phi$ satisfying the following conditions:

(a) $\phi$ is a positive increasing function on $(0, \infty)$ such that $t^{\delta} \phi^{\prime}(t)$ is monotonic on $(0, \infty)$ for some $\delta \in \mathbb{R}$;

(b) There exist positive constants $C_{\phi}$ and $c_{\phi}$ such that $t \phi^{\prime}(t) \geq C_{\phi} \phi(t)$ and $\phi(2 t) \leq c_{\phi} \phi(t)$ for all $t>0$.

It is clear that $\mathfrak{F}_{3} \subsetneq \mathfrak{F}_{1}$. There are some model examples for the class $\mathfrak{F}_{3}$ such as $t^{\alpha}(\alpha>0)$, $t^{\beta} \ln (1+t)(\beta \geq 1), t \ln \ln (e+t)$, real-valued polynomials $P$ on $\mathbb{R}$ with positive coefficients and $P(0)=0$ and so on.

Based on the above, it is natural to ask the following question.

Question 1.3 Is $T_{h, \Omega, P, \varphi}^{*}$ defined in (1.4) bounded on the Triebel-Lizorkin spaces if $h \equiv 1$ and $\Omega \in H^{1}\left(\mathrm{~S}^{n-1}\right)$ ?

Our next result will give a positive answer to Question 1.3.

Theorem 1.3 Let $P$ be a real polynomial on $\mathbb{R}$ satisfying $P(0)=0$ and $\varphi \in \mathfrak{F}_{3}$. Suppose that $h \equiv 1$ and $\Omega \in H^{1}\left(S^{n-1}\right)$ satisfies (1.1). Then, for $0<\alpha<1$ and $1<p, q<\infty$, there exists a constant $C>0$ independent of $\Omega$ and the coefficients of $P$ such that

$$
\begin{array}{ll}
\left\|T_{h, \Omega, P, \varphi}^{*} f\right\|_{\dot{F}_{\alpha}^{p, q}\left(\mathbb{R}^{n}\right)} \leq C\|\Omega\|_{H^{1}\left(S^{n-1}\right)}\|f\|_{\dot{F}_{\alpha}^{p, q}\left(\mathbb{R}^{n}\right)}, & \forall f \in \dot{F}_{\alpha}^{p, q}\left(\mathbb{R}^{n}\right) ; \\
\left\|T_{h, \Omega, P, \varphi}^{*} f\right\|_{F_{\alpha}^{p, q}\left(\mathbb{R}^{n)}\right.} \leq C\|\Omega\|_{H^{1}\left(S^{n-1}\right)}\|f\|_{F_{\alpha}^{p, q}\left(\mathbb{R}^{n}\right)}, & \forall f \in F_{\alpha}^{p, q}\left(\mathbb{R}^{n}\right) .
\end{array}
$$

Moreover, both $T_{h, \Omega, P, \varphi}^{*}: F_{\alpha}^{p, q}\left(\mathbb{R}^{n}\right) \rightarrow \dot{F}_{\alpha}^{p, q}\left(\mathbb{R}^{n}\right)$ and $T_{h, \Omega, P, \varphi}^{*}: F_{\alpha}^{p, q}\left(\mathbb{R}^{n}\right) \rightarrow F_{\alpha}^{p, q}\left(\mathbb{R}^{n}\right)$ are continuous.

Remark 1.4 The boundedness part in Theorem 1.3 implies [43, Theorem 1.2] when $P(t)=$ $\varphi(t) \equiv t$. It should be pointed out that Theorem 1.3 is new, even in the special case $\varphi(t) \equiv t$.

The paper is organized as follows. In Sect. 2 we present some preliminary definitions and lemmas, which are the main ingredients of proving Theorems 1.1-1.3. The proofs of Theorems 1.1-1.3 will be given in Sect. 3. It should be pointed out that the main methods and ideas employed in this paper are a combination of ideas and arguments from [1, 21, $22,27,30,41]$. However, some new techniques are needed in the main proofs. The new ideas invented in our proofs are to define suitable measures and to estimate them suitably.

Throughout the paper, for any $p \in[1, \infty]$, we denote $p^{\prime}$ by the conjugate index of $p$, which satisfies $1 / p+1 / p^{\prime}=1$. Here, we set $1^{\prime}=\infty$ and $\infty^{\prime}=1$. The letter $C$ or $c$, sometimes with certain parameters, will stand for positive constants not necessarily the same one at each occurrence, but are independent of the essential variables. In what follows, we set $\mathfrak{R}_{n}=\left\{\zeta \in \mathbb{R}^{n} ; 1 / 2<|\zeta| \leq 1\right\}$. Let $\triangle_{\zeta}(f)$ be the difference of $f$ for an arbitrary function $f$ defined on $\mathbb{R}^{n}$ and $\zeta \in \mathbb{R}^{n}$, i.e., $\triangle_{\zeta}(f)(x)=f_{\zeta}(x)-f(x)$, where $f_{\zeta}(x)=f(x+\zeta)$. For any $t \in \mathbb{R}$, we set $\exp (t)=e^{-2 \pi i t}$. We also use the conventions $\sum_{i \in \emptyset} a_{i}=0$ and $\prod_{i \in \emptyset} a_{i}=1$. 


\section{Preliminary definitions and lemmas}

\subsection{Preliminary definitions}

In this subsection we give the definitions of several rough kernels and their relationships.

Definition 2.1 (Hardy spaces) The Hardy space $H^{1}\left(S^{n-1}\right)$ is the set of all $L^{1}\left(S^{n-1}\right)$ functions which satisfy $\|f\|_{H^{1}\left(S^{n-1}\right)}<\infty$, where

$$
\|\Omega\|_{H^{1}\left(S^{n-1}\right)}:=\int_{S^{n-1}} \sup _{0 \leq r<1}\left|\int_{S^{n-1}} \Omega(\theta) \frac{1-r^{2}}{|r w-\theta|^{n}} d \sigma(\theta)\right| d \sigma(w) .
$$

Definition 2.2 $\left(L(\log L)^{\alpha}\left(\mathrm{S}^{n-1}\right)\right.$ class) The class $L(\log L)^{\alpha}\left(\mathrm{S}^{n-1}\right)$ for $\alpha>0$ denotes the class of all measurable functions $\Omega$ on $S^{n-1}$ which satisfy

$$
\|\Omega\|_{L(\log L)^{\alpha}\left(S^{n-1}\right)}:=\int_{S^{n-1}}|\Omega(\theta)| \log ^{\alpha}(|\Omega(\theta)|+2) d \sigma(\theta)<\infty .
$$

Definition 2.3 (Grafakos-Stefanov class) The Grafakos-Stefanov class $\mathcal{F}_{\beta}\left(\mathrm{S}^{n-1}\right)$ for $\beta>0$ denotes the set of all integrable functions over $S^{n-1}$ which satisfy the condition

$$
\sup _{u \in S^{n-1}} \int_{S^{n-1}}|\Omega(v)|\left(\log ^{+} \frac{1}{|u \cdot v|}\right)^{\beta} d \sigma(v)<\infty
$$

We remark that $\mathcal{F}_{\beta}\left(\mathrm{S}^{n-1}\right)$ was introduced by Grafakos and Stefanov [25] in the study of the $L^{p}$ boundedness of singular integral operator with rough kernels.

The following inclusion relations are known:

$$
\begin{aligned}
& L^{r}\left(\mathrm{~S}^{n-1}\right) \subsetneq L(\log L)^{\beta_{1}}\left(\mathrm{~S}^{n-1}\right) \subsetneq L(\log L)^{\beta_{2}}\left(\mathrm{~S}^{n-1}\right) \quad \text { for } r>1 \text { and } 0<\beta_{2}<\beta_{1} ; \\
& L(\log L)^{\beta}\left(\mathrm{S}^{n-1}\right) \subsetneq H^{1}\left(\mathrm{~S}^{n-1}\right) \subsetneq L^{1}\left(\mathrm{~S}^{n-1}\right) \quad \text { for } \beta \geq 1 ; \\
& L(\log L)^{\beta}\left(\mathrm{S}^{n-1}\right) \nsubseteq H^{1}\left(\mathrm{~S}^{n-1}\right) \nsubseteq L(\log L)^{\beta}\left(\mathrm{S}^{n-1}\right) \quad \text { for } 0<\beta<1 ; \\
& \mathcal{F}_{\beta_{1}}\left(\mathrm{~S}^{n-1}\right) \subsetneq \mathcal{F}_{\beta_{2}}\left(\mathrm{~S}^{n-1}\right), \quad 0<\beta_{2}<\beta_{1} ; \\
& \bigcup_{q>1} L^{q}\left(\mathrm{~S}^{n-1}\right) \subsetneq \mathcal{F}_{\beta}\left(\mathrm{S}^{n-1}\right), \quad \beta>0 ; \\
& \bigcap_{\beta>1} \mathcal{F}_{\beta}\left(\mathrm{S}^{n-1}\right) \nsubseteq H^{1}\left(\mathrm{~S}^{n-1}\right) \nsubseteq \bigcup_{\beta>1} \mathcal{F}_{\beta}\left(\mathrm{S}^{n-1}\right) .
\end{aligned}
$$

Let us present the definitions of Triebel-Lizorkin spaces.

Definition 2.4 (Triebel-Lizorkin spaces) Let $\mathcal{S}^{\prime}\left(\mathbb{R}^{n}\right)$ be the tempered distribution class on $\mathbb{R}^{n}$. For $\alpha \in \mathbb{R}$ and $0<p, q \leq \infty(p \neq \infty)$, we define the homogeneous Triebel-Lizorkin spaces $\dot{F}_{\alpha}^{p, q}\left(\mathbb{R}^{n}\right)$ by

$$
\dot{F}_{\alpha}^{p, q}\left(\mathbb{R}^{n}\right):=\left\{f \in \mathcal{S}^{\prime}\left(\mathbb{R}^{n}\right):\|f\|_{\dot{F}_{\alpha}^{p, q}\left(\mathbb{R}^{n}\right)}=\left\|\left(\sum_{i \in \mathbb{Z}} 2^{-i \alpha q}\left|\Psi_{i} * f\right|^{q}\right)^{1 / q}\right\|_{L^{p}\left(\mathbb{R}^{n}\right)}<\infty\right\}
$$

where $\widehat{\Psi}_{i}(\xi)=\phi\left(2^{i} \xi\right)$ for $i \in \mathbb{Z}$ and $\phi \in \mathcal{C}_{c}^{\infty}\left(\mathbb{R}^{n}\right)$ satisfies the conditions: $0 \leq \phi(x) \leq 1$; $\operatorname{supp}(\phi) \subset\{x: 1 / 2 \leq|x| \leq 2\} ; \phi(x)>c>0$ if $3 / 5 \leq|x| \leq 5 / 3$. The inhomogeneous versions 
of Triebel-Lizorkin spaces are denoted by $F_{\alpha}^{p, q}\left(\mathbb{R}^{n}\right)$ and are obtained by adding the term $\|\Theta * f\|_{L^{p}\left(\mathbb{R}^{n}\right)}$ to the right-hand side of (2.3) with $\sum_{i \in \mathbb{Z}}$ replaced by $\sum_{i \geq 1}$, where $\Theta \in \mathcal{S}\left(\mathbb{R}^{n}\right)$, $\operatorname{supp}(\hat{\Theta}) \subset\{\xi:|\xi| \leq 2\}, \hat{\Theta}(x)>c>0$ if $|x| \leq 5 / 3$.

The following properties are well known (see $[24,40])$ :

$$
\begin{aligned}
& \dot{F}_{0}^{p, 2}\left(\mathbb{R}^{n}\right)=L^{p}\left(\mathbb{R}^{n}\right) \quad \text { for } 1<p<\infty ; \\
& F_{\alpha}^{p, q}\left(\mathbb{R}^{n}\right) \sim \dot{F}_{\alpha}^{p, q}\left(\mathbb{R}^{n}\right) \cap L^{p}\left(\mathbb{R}^{n}\right) \quad \text { and } \\
& \|f\|_{F_{\alpha}^{p, q}\left(\mathbb{R}^{n}\right)} \sim\|f\|_{\dot{F}_{\alpha}^{p, q}\left(\mathbb{R}^{n}\right)}+\|f\|_{L^{p}\left(\mathbb{R}^{n}\right)} \quad \text { for } \alpha>0,1<p, q<\infty .
\end{aligned}
$$

Our next definition is concerned with the $H^{1}\left(\mathrm{~S}^{n-1}\right)$ atom.

Definition $2.5\left(H^{1}\left(\mathrm{~S}^{n-1}\right)\right.$ atom) A function $a: \mathrm{S}^{n-1} \rightarrow \mathbb{C}$ is a $(1, \infty)$ atom if there exist $\vartheta \in \mathrm{S}^{n-1}$ and $\varrho \in(0,1]$ such that

$$
\begin{aligned}
& \operatorname{supp}(a) \subset S^{n-1} \cap B(\vartheta, \varrho), \quad \text { where } B(\vartheta, \varrho)=\left\{y \in \mathbb{R}^{n}:|y-\vartheta|<\varrho\right\} ; \\
& \|a\|_{L^{\infty}\left(S^{n-1}\right)} \leq \varrho^{-n+1} ; \\
& \int_{S^{n-1}} a(y) d \sigma(y)=0 .
\end{aligned}
$$

\subsection{Preliminary lemmas}

We start now the following atomic decomposition of $H^{1}\left(\mathrm{~S}^{n-1}\right)$.

Lemma 2.1 ([13,14]) Let $\Omega \in H^{1}\left(\mathrm{~S}^{n-1}\right)$ satisfy $(1.1)$. Then there exist a sequence of complex numbers $\left\{c_{j}\right\}_{j \geq 1}$ and a sequence of $(1, \infty)$ atoms $\left\{\Omega_{j}\right\}_{j \geq 1}$ such that

$$
\Omega=\sum_{j=1}^{\infty} c_{j} \Omega_{j}, \quad\|\Omega\|_{H^{1}\left(S^{n-1)}\right)} \approx \sum_{j=1}^{\infty}\left|c_{j}\right| .
$$

In order to deal with certain estimates for Fourier transforms of some measures, we need the following properties for $(1, \infty)$ atom.

Lemma 2.2 ([21]) Let $\zeta=\left(\zeta_{1}, \ldots, \zeta_{n}\right) \neq(0, \ldots, 0)$ and $\zeta^{\prime}=\zeta /|\zeta|=\left(\zeta_{1}^{\prime}, \ldots, \zeta_{n}^{\prime}\right)$. Suppose that $n \geq 3$ and $b(\cdot)$ is a $(1, \infty)$ atom on $\mathrm{S}^{n-1}$ supported in $\mathrm{S}^{n-1} \cap B\left(\zeta^{\prime}, \varrho\right)$, where $\varrho \in(0,1]$. Let

$$
\begin{aligned}
& F_{b}(s)=\left(1-s^{2}\right)^{(n-3) / 2} \chi_{(-1,1)}(s) \int_{S^{n-2}} b\left(s,\left(1-s^{2}\right)^{1 / 2} \tilde{y}\right) d \sigma(\tilde{y}), \\
& G_{b}(s)=\left(1-s^{2}\right)^{(n-3) / 2} \chi_{(-1,1)}(s) \int_{S^{n-2}}\left|b\left(s,\left(1-s^{2}\right)^{1 / 2} \tilde{y}\right)\right| d \sigma(\tilde{y}) .
\end{aligned}
$$

Then there exists a positive constant $C$, independent of $b$, such that

$$
\begin{aligned}
& \operatorname{supp}\left(F_{b}\right) \subset\left(\zeta_{1}^{\prime}-2 r\left(\zeta^{\prime}\right), \zeta_{1}^{\prime}+2 r\left(\zeta^{\prime}\right)\right), \\
& \operatorname{supp}\left(G_{b}\right) \subset\left(\zeta_{1}^{\prime}-2 r\left(\zeta^{\prime}\right), \zeta_{1}^{\prime}+2 r\left(\zeta^{\prime}\right)\right) ; \\
& \left\|F_{b}\right\|_{L^{\infty}(\mathbb{R})} \leq C\left|r\left(\zeta^{\prime}\right)\right|^{-1}, \quad\left\|G_{b}\right\|_{L^{\infty}(\mathbb{R})} \leq C\left|r\left(\zeta^{\prime}\right)\right|^{-1} ;
\end{aligned}
$$




$$
\int_{\mathbb{R}} F_{b}(s) d s=0
$$

where $r\left(\zeta^{\prime}\right)=|\zeta|^{-1}\left|A_{\varrho}(\zeta)\right|$ and $A_{\varrho}(\zeta)=\left(\varrho^{2} \zeta_{1}, \varrho \zeta_{2}, \ldots, \varrho \zeta_{n}\right)$.

Lemma 2.3 ([21]) Let $\zeta=\left(\zeta_{1}, \zeta_{2}\right) \neq(0,0)$ and $\zeta^{\prime}=\zeta /|\zeta|=\left(\zeta_{1}^{\prime}, \zeta_{2}^{\prime}\right)$. Suppose that $n=2$ and $b(\cdot)$ is a $(1, \infty)$ atom on $\mathrm{S}^{1}$ supported in $\mathrm{S}^{1} \cap B\left(\zeta^{\prime}, \varrho\right)$, where $\varrho \in(0,1]$. Let

$$
\begin{aligned}
& F_{b}(s)=\left(1-s^{2}\right)^{-1 / 2} \chi_{(-1,1)}(s)\left(b\left(s,\left(1-s^{2}\right)^{1 / 2}\right)+b\left(s,-\left(1-s^{2}\right)^{1 / 2}\right)\right), \\
& G_{b}(s)=\left(1-s^{2}\right)^{-1 / 2} \chi_{(-1,1)}(s)\left(\left|b\left(s,\left(1-s^{2}\right)^{1 / 2}\right)\right|+\left|b\left(s,-\left(1-s^{2}\right)^{1 / 2}\right)\right|\right) .
\end{aligned}
$$

Then there exists a positive constant $C$, independent of $b$, such that

$$
\begin{aligned}
& \operatorname{supp}\left(F_{b}\right) \subset\left(\zeta_{1}^{\prime}-2 r\left(\zeta^{\prime}\right), \zeta_{1}^{\prime}+2 r\left(\zeta^{\prime}\right)\right), \quad \operatorname{supp}\left(G_{b}\right) \subset\left(\zeta_{1}^{\prime}-2 r\left(\zeta^{\prime}\right), \zeta_{1}^{\prime}+2 r\left(\zeta^{\prime}\right)\right) ; \\
& \int_{\mathbb{R}} F_{b}(s) d s=0 ; \\
& \left\|F_{b}\right\|_{L^{q}(\mathbb{R})} \leq C\left|r\left(\zeta^{\prime}\right)\right|^{-1+1 / q}, \quad\left\|G_{b}\right\|_{L^{q}(\mathbb{R})} \leq C\left|r\left(\zeta^{\prime}\right)\right|^{-1+1 / q}, \\
& \text { for some } q \in(1,2) \text {, where } r\left(\zeta^{\prime}\right)=|\zeta|^{-1}\left|A_{\varrho}(\zeta)\right| \text { and } A_{\varrho}(\zeta)=\left(\varrho^{2} \zeta_{1}, \varrho \zeta_{2}\right) .
\end{aligned}
$$

The following oscillatory estimates are useful for our proofs.

Lemma 2.4 ([33, Corollary, p. 186]) Let $l \in \mathbb{N} \backslash\{0\},\left\{\mu_{i}\right\}_{i=1}^{l} \subset \mathbb{R}$, and $\left\{d_{i}\right\}_{i=1}^{l}$ be distinct positive real numbers. Let $\psi \in \mathcal{C}^{1}([0,1])$. Then there exists $C>0$ independent of $\left\{\mu_{j}\right\}_{j=1}^{l}$ such that

$$
\left|\int_{\delta}^{\tau} \exp \left(\mu_{1} t^{d_{1}}+\cdots+\mu_{l} t^{d_{l}}\right) \psi(t) d t\right| \leq C\left|\mu_{1}\right|^{-\epsilon}\left(|\psi(\tau)|+\int_{\delta}^{\tau}\left|\psi^{\prime}(t)\right| d t\right)
$$

holds for $0 \leq \delta<\tau \leq 1$ and $\epsilon=\min \left\{1 / d_{1}, 1 / l\right\}$.

Lemma 2.5 ([31]) Let $\Phi(t)=t^{\alpha_{1}}+\mu_{2} t^{\alpha_{2}}+\cdots+\mu_{n} t^{\alpha_{n}}$, where $\left\{\mu_{i}\right\}_{i=2}^{n}$ are real parameters, and $\left\{\alpha_{i}\right\}_{i=1}^{n}$ are distinct positive (not necessarily integer) exponents. Suppose that $\varphi \in \mathfrak{F}_{3}$ and $t^{\delta} \varphi^{\prime}(t)$ is monotonic on $(0, \infty)$ for some $\delta \in \mathbb{R}$. Then, for any $r>0$ and $\lambda \neq 0$,

$$
\left|\int_{r / 2}^{r} \exp (\lambda \Phi(\varphi(t))) \frac{d t}{t}\right| \leq C\left|\lambda \varphi(r)^{\alpha_{1}}\right|^{-\epsilon}
$$

with $\epsilon=\min \left\{1 / \alpha_{1}, 1 / n\right\}$. Here, $C>0$ is independent of $\left\{\mu_{i}\right\}_{i=2}^{n}$, but may depend on $\varphi$ and $\delta$.

We end this section by presenting a well-known result.

Lemma 2.6 ([38, pp. 476-478]) Let $\mathcal{P}=\left(P_{1}, \ldots, P_{d}\right)$ with each $P_{i}$ being a real polynomial defined on $\mathbb{R}^{n}$. Then the maximal operator $M_{\mathcal{P}}$ defined by

$$
M_{\mathcal{P}} f(x)=\sup _{r>0} \frac{1}{r^{n}}\left|\int_{|t| \leq r} f(x-\mathcal{P}(t)) d t\right|
$$


satisfies

$$
\left\|M_{\mathcal{P}} f\right\|_{L^{p}\left(\mathbb{R}^{d}\right)} \leq C_{p}\|f\|_{L^{p}\left(\mathbb{R}^{d}\right)}, \quad \forall 1<p<\infty \text { and } f \in L^{p}\left(\mathbb{R}^{d}\right) .
$$

Here, $C_{p}>0$ is independent of the coefficients of $\left\{P_{i}\right\}_{i=1}^{d}$ and $f$.

\section{Proofs of Theorems 1.1-1.3}

In this section we prove Theorems 1.1-1.3. In Sect. 3.1 we present some notation and lemmas, which are the main ingredients of proving Theorems 1.1-1.3. The proofs of Theorems 1.1-1.3 will be given in Sect. 3.2.

\subsection{Some notation and lemmas}

In what follows, let $N \in \mathbb{N} \backslash\{0\}$ and $P(t)=\sum_{i=1}^{N} a_{i} t^{i}$ with $a_{N} \neq 0$. Then there exist $0<l_{1}<$ $l_{2}<\cdots<l_{\Lambda}=N$ such that $P(t)=\sum_{i=1}^{\Lambda} a_{l_{i}} t^{l_{i}}$ with $a_{l_{i}} \neq 0$ for all $1 \leq i \leq \Lambda$. Set

$$
P_{0}(t)=0, \quad P_{s}(t)=\sum_{i=1}^{s} a_{l_{s}} t^{l_{s}}, \quad 1 \leq s \leq \Lambda .
$$

It is clear that $P(t)=P_{\Lambda}(t)$ and $l_{s} \geq s$ for $1 \leq s \leq \Lambda$.

Let $h, \Omega$ be given as in (1.2). For $0 \leq s \leq \Lambda, y, \xi \in \mathbb{R}^{n}$, a vector $\theta \in \mathrm{S}^{n-1}$, and a function $\varphi:[0, \infty) \rightarrow \mathbb{R}$, we set

$$
\Gamma_{s, \theta}(y, \xi)=\sum_{i=s+1}^{\Lambda} a_{l_{i}} \varphi(|y|)^{l_{i}} \theta \cdot \xi
$$

Define the measures $\left\{\sigma_{h, \Omega, k, \theta, s}\right\}_{k \in \mathbb{Z}}$ and $\left\{\left|\sigma_{h, \Omega, k, \theta, s}\right|\right\}_{k \in \mathbb{Z}}$ by

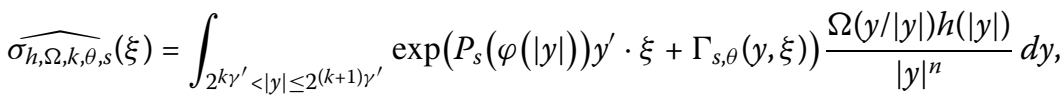

$$
\begin{aligned}
& \left|\widehat{\sigma_{h, \Omega, k, \theta, s}}\right|(\xi)=\int_{2^{k \gamma^{\prime}}<|y| \leq 2^{(k+1) \gamma^{\prime}}} \exp \left(P_{s}(\varphi(|y|)) y^{\prime} \cdot \xi+\Gamma_{s, \theta}(y, \xi)\right) \frac{|\Omega(y /|y|) h(|y|)|}{|y|^{n}} d y,
\end{aligned}
$$

where $P_{s}$ is given as in (3.1). Note that $\Gamma_{s, \theta}(y, \xi)$ is independent of $y /|y|$. In view of (1.1), it is easy to see that

$$
\sigma_{h, \Omega, k, \theta, 0}(\xi)=0, \quad \forall k \in \mathbb{Z}, \xi \in \mathbb{R}^{n} .
$$

We have the following estimates.

Lemma 3.1 Let $h \in \Delta_{\gamma}\left(\mathbb{R}_{+}\right)$for some $\gamma \in(1, \infty]$ and $\Omega$ be a $(1, \infty)$ atom satisfying (2.6)(2.8) with $0<\varrho \leq 1$ and $\vartheta=\theta=(1,0, \ldots, 0) \in \mathrm{S}^{n-1}$. Assume that $\varphi \in \mathfrak{F}_{1}$ or $\varphi \in \mathfrak{F}_{2}$. Then, for $1 \leq s \leq \Lambda$ and $\xi=\left(\xi_{1}, \ldots, \xi_{n}\right) \neq(0, \ldots, 0)$, there exists a constant $C>0$ independent of $h, \Omega, \gamma, \xi$ and $\left\{a_{l_{s}}\right\}_{s=1}^{\Lambda}$ such that

$$
\left|\widehat{\sigma_{h, \Omega, k, \theta, s}}(\xi)-\widehat{\sigma_{h, \Omega, k, \theta, s-1}}(\xi)\right| \leq C \gamma^{\prime}\|h\|_{\Delta_{\gamma}\left(\mathbb{R}_{+}\right)} \min \left\{1, \varphi\left(2^{(k+1) \gamma^{\prime}}\right)^{l_{s}}\left|L_{s}(\xi)\right|\right\}
$$


where

$$
L_{s}(\xi)=\left(a_{l_{s}} \varrho^{2} \xi_{1}, a_{l_{s}} \varrho \xi_{2}, \ldots, a_{l_{s}} \varrho \xi_{n}\right) .
$$

Proof We only prove (3.3) for the case $\varphi \in \mathfrak{F}_{1}$ since another case $\varphi \in \mathfrak{F}_{2}$ is analogous. Fix $1 \leq s \leq \Lambda$ and $\xi^{\prime}=\xi /|\xi|=\left(\xi_{1}^{\prime}, \ldots, \xi_{n}^{\prime}\right)$. Let $\mathcal{O}$ be the rotation such that $\mathcal{O}\left(\xi^{\prime}\right)=\vartheta$ and $\mathcal{O}^{-1}$ denote the inverse of $\mathcal{O}$. Then $\mathcal{O}^{2}\left(\xi^{\prime}\right)=\left(\xi_{1}^{\prime}, \eta_{2}^{\prime}, \ldots, \eta_{n}^{\prime}\right)$. Let $Q_{n-1}$ be a rotation in $\mathbb{R}^{n-1}$ such that $Q_{n-1}\left(\xi_{2}^{\prime}, \ldots, \xi_{n}^{\prime}\right)=\left(\eta_{2}^{\prime}, \ldots, \eta_{n}^{\prime}\right)$ and $R$ be a transformation by $R\left(z_{1}, z_{2}, \ldots, z_{n}\right)=$ $\left(z_{1}, Q_{n-1}\left(z_{2}, \ldots, z_{n}\right)\right)$. Then, for any $y^{\prime}=\left(u, y_{2}^{\prime}, \ldots, y_{n}^{\prime}\right) \in S^{n-1}$, we have $\vartheta \cdot R\left(y^{\prime}\right)=\vartheta \cdot y^{\prime}=u$ and $\Omega\left(\mathcal{O}^{-1} R\left(y^{\prime}\right)\right)$ is a $(1, \infty)$ atom with supported in $S^{n-1} \cap B\left(\xi^{\prime}, \varrho\right)$. By some changes of variables, we have

$$
\begin{aligned}
& \widehat{\sigma_{h, \Omega, k, \theta}, s}(\xi) \\
& =\int_{2^{k \gamma^{\prime}}}^{2^{(k+1) \gamma^{\prime}}} \exp \left(\sum_{i=s+1}^{\Lambda} a_{l_{i}} \varphi(t)^{l_{i}} \xi \cdot \theta\right) \int_{S^{n-1}} \Omega\left(y^{\prime}\right) \exp \left(\sum_{i=1}^{s} a_{l_{i}} \varphi(t)^{l_{i}} \xi \cdot y^{\prime}\right) d \sigma\left(y^{\prime}\right) h(t) \frac{d t}{t} \\
& =\int_{2^{k \gamma^{\prime}}}^{2^{(k+1) \gamma^{\prime}}} \exp \left(\sum_{i=s+1}^{\Lambda} a_{l_{i}} \varphi(t)^{l_{i}}|\xi| \xi_{1}^{\prime}\right) \\
& \times \int_{S^{n-1}} A\left(y^{\prime}\right) \exp \left(\sum_{i=1}^{s} a_{l_{i}} \varphi(t)^{l_{i}}|\xi| \xi^{\prime} \cdot \mathcal{O}^{-1} R\left(y^{\prime}\right)\right) d \sigma\left(y^{\prime}\right) h(t) \frac{d t}{t} \\
& =\int_{2^{k \gamma^{\prime}}}^{2^{(k+1) \gamma^{\prime}}} \exp \left(\sum_{i=s+1}^{\Lambda} a_{l_{i}} \varphi(t)^{l_{i}}|\xi| \xi_{1}^{\prime}\right) \int_{\mathbb{R}} F_{A}(u) \exp \left(\sum_{i=1}^{s} a_{l_{i}} \varphi(t)^{l_{i}}|\xi| u\right) d u h(t) \frac{d t}{t}
\end{aligned}
$$

where $A\left(y^{\prime}\right)=\Omega\left(\mathcal{O}^{-1} R\left(y^{\prime}\right)\right)$ and $F_{A}$ is defined as in Lemma 2.2 (in case $n>2$ ) or Lemma 2.3 (in case $n=2)$. Notice that $A(\cdot)$ is a $(1, \infty)$ atom with supported in $B\left(\xi^{\prime}, \varrho\right)$. Invoking Lemmas 2.2 and 2.3 , one finds that

$$
\begin{aligned}
& \operatorname{supp}\left(F_{A}\right) \subset\left(\xi_{1}^{\prime}-2 r\left(\xi^{\prime}\right), \xi_{1}^{\prime}+2 r\left(\xi^{\prime}\right)\right) . \\
& \left\|F_{A}\right\|_{L^{\infty}(\mathbb{R})} \leq C\left|r\left(\xi^{\prime}\right)\right|^{-1}, \quad \text { if } n \geq 3 ; \\
& \left\|F_{A}\right\|_{L^{q}(\mathbb{R})} \leq C\left|r\left(\xi^{\prime}\right)\right|^{-1+1 / q}, \quad \text { if } n=2
\end{aligned}
$$

for some $q \in(1,2)$. Here, $r\left(\xi^{\prime}\right)=|\xi|^{-1} L_{\varrho}(\xi)$, where $A_{\varrho}(\xi)=\left(\varrho^{2} \xi_{1}, \varrho \xi_{2}, \ldots, \varrho \xi_{n}\right)$ for $n \geq 3$ and $A_{\varrho}(\xi)=\left(\varrho^{2} \xi_{1}, \varrho \xi_{2}\right)$ for $n=2$.

In view of (3.5) and (3.6),

$$
\begin{aligned}
& \left|\widehat{\sigma_{h, \Omega, k, \theta, s}}(\xi)-\widehat{\sigma_{h, \Omega, k, \theta, s-1}}(\xi)\right| \\
& =\mid \int_{2^{k \gamma^{\prime}}}^{2^{(k+1) \gamma^{\prime}}} \exp \left(\sum_{i=s+1}^{\Lambda} a_{l_{i}} \varphi(t)^{l_{i}}|\xi| \xi_{1}^{\prime}\right) \int_{\mathbb{R}} F_{A}(u) \exp \left(\sum_{i=1}^{s-1} a_{l_{i}} \varphi(t)^{l_{i}}|\xi| u\right) \\
& \quad \times\left(\exp \left(a_{l_{s}} \varphi(t)^{l_{s}}|\xi| \xi_{1}^{\prime}\right)-\exp \left(a_{l_{s}} \varphi(t)^{l_{s}}|\xi| u\right)\right) d u h(t) \frac{d t}{t} \mid \\
& \leq \int_{2^{k \gamma^{\prime}}}^{2^{(k+1) \gamma^{\prime}}} \int_{\mathbb{R}}\left|F_{A}(u)\right| \min \left\{2,2 \pi \varphi\left(2^{(k+1) \gamma^{\prime}}\right)^{l_{s}}\left|a_{l_{s}} \xi\right|\left|\xi_{1}^{\prime}-u\right|\right\} d u|h(t)| \frac{d t}{t}
\end{aligned}
$$




$$
\leq \min \left\{2,4 \pi\left|a_{l_{s}} \xi\right| r\left(\xi^{\prime}\right) \varphi\left(2^{(k+1) \gamma^{\prime}}\right)^{l_{s}}\right\} \int_{2^{k \gamma^{\prime}}}^{2^{(k+1) \gamma^{\prime}}}|h(t)| \frac{d t}{t} \int_{\mathbb{R}}\left|F_{A}(u)\right| d u .
$$

From (3.7) and (3.8), one sees that there exists $C>0$ independent of $h, \Omega, \gamma$ such that

$$
\int_{\mathbb{R}}\left|F_{A}(u)\right| d u \leq C .
$$

Moreover, by Hölder's inequality, one has

$$
\begin{aligned}
\int_{2^{k \gamma^{\prime}}}^{2^{(k+1) \gamma^{\prime}}}|h(t)| \frac{d t}{t} & =\sum_{i=0}^{\left[\gamma^{\prime}\right]} \int_{2^{k \gamma^{\prime}+i}}^{2^{k \gamma^{\prime}+i+1}}|h(t)| \frac{d t}{t} \\
& \leq \sum_{i=0}^{\left[\gamma^{\prime}\right]}\left(\int_{2^{k \gamma^{\prime}+i}}^{2^{k \gamma^{\prime}+i+1}}|h(t)|^{\gamma} \frac{d t}{t}\right)^{1 / \gamma}\left(\int_{2^{k \gamma^{\prime}+i}}^{2^{k \gamma^{\prime}+i+1}} \frac{d t}{t}\right)^{1 / \gamma^{\prime}} \\
& \leq 2^{1 / \gamma}\left(\left[\gamma^{\prime}\right]+1\right)\|h\|_{\Delta_{\gamma}\left(\mathbb{R}_{+}\right)}(\ln 2)^{1 / \gamma^{\prime}} \leq 4 \gamma^{\prime}\|h\|_{\Delta_{\gamma}\left(\mathbb{R}_{+}\right)} .
\end{aligned}
$$

Here, $[x]=\max \{k \in \mathbb{Z}: k \leq x\}$ for $x \in \mathbb{R}$. Finally, it follows from (3.9)-(3.11) that

$$
\left|\widehat{\sigma_{h, \Omega, k, \theta, s}}(\xi)-\widehat{\sigma_{h, \Omega, k, \theta, s-1}}(\xi)\right| \leq C \gamma^{\prime}\|h\|_{\Delta_{\gamma}\left(\mathbb{R}_{+}\right)} \min \left\{1, \varphi\left(2^{(k+1) \gamma^{\prime}}\right)^{l_{s}}\left|L_{s}(\xi)\right|\right\}
$$

where $C>0$ is independent of $h, \Omega, \gamma$. This proves (3.3) and completes the proof.

Lemma 3.2 Let $h \in \Delta_{\gamma}\left(\mathbb{R}_{+}\right)$for some $\gamma \in(1, \infty]$ and $\Omega$ be a $(1, \infty)$ atom satisfying $(2.6)$ (2.8) with $0<\varrho \leq 1$ and $\vartheta=\theta=(1,0, \ldots, 0) \in \mathrm{S}^{n-1}$. Assume that $\varphi \in \mathfrak{F}_{1}$ or $\varphi \in \mathfrak{F}_{2}$. Then, for $1 \leq s \leq \Lambda$ and $\xi=\left(\xi_{1}, \ldots, \xi_{n}\right) \neq(0, \ldots, 0)$, there exist $\delta>0$ and $C>0$ independent of $h, \Omega, \gamma, \xi$, and $\left\{a_{l_{s}}\right\}_{s=1}^{\Lambda}$ such that

$$
\left|\widehat{\sigma_{h, \Omega, k, \theta, s}}(\xi)\right| \leq C \gamma^{\prime}\|h\|_{\Delta_{\gamma}\left(\mathbb{R}_{+}\right)} \min \left\{1,\left(\varphi\left(2^{k \gamma^{\prime}}\right)^{l_{s}}\left|L_{s}(\xi)\right|\right)^{-1 /\left(2 l_{\gamma} \gamma^{\prime} \delta\right)}\right\},
$$

where $L_{s}(\xi)$ is given as (3.4) and $\delta=1$ if $n \geq 3$ and $\delta>2$ if $n=2$.

Proof We only prove (3.12) for the case $\varphi \in \mathfrak{F}_{1}$ since another case is analogous. By (3.5) and Hölder's inequality, we have

$$
\begin{aligned}
& \left|\widehat{\sigma_{h, \Omega, k, \theta, s}}(\xi)\right| \\
& \leq \int_{2^{k \gamma^{\prime}}}^{2^{(k+1) \gamma^{\prime}}}\left|\int_{\mathbb{R}} F_{A}(u) \exp \left(\sum_{i=1}^{s} a_{l_{i}} \varphi(t)^{l_{i}}|\xi| u\right) d u\right||h(t)| \frac{d t}{t} \\
& \leq\left(\int_{2^{k \gamma^{\prime}}}^{2^{(k+1) \gamma^{\prime}}}|h(t)|^{\gamma} \frac{d t}{t}\right)^{1 / \gamma}\left(\int_{2^{k \gamma^{\prime}}}^{2^{(k+1) \gamma^{\prime}}}\left|\int_{\mathbb{R}} F_{A}(u) \exp \left(\sum_{i=1}^{s} a_{l_{i}} \varphi(t)^{l_{i}}|\xi| u\right) d u\right|^{\gamma^{\prime}} \frac{d t}{t}\right)^{1 / \gamma^{\prime}} \\
& \leq\left(\int_{2^{k \gamma^{\prime}}}^{2^{(k+1) \gamma^{\prime}}}|h(t)|^{\gamma} \frac{d t}{t}\right)^{1 / \gamma}\left\|F_{A}\right\|_{L^{1}(\mathbb{R})}^{\max \left\{1-2 / \gamma^{\prime}, 0\right\}}\left(\gamma^{\prime}\right)^{\max \left\{1 / \gamma^{\prime}-1 / 2,0\right\}} \\
& \times\left(\int_{2^{k \gamma^{\prime}}}^{2^{(k+1) \gamma^{\prime}}}\left|\int_{\mathbb{R}} F_{A}(u) \exp \left(\sum_{i=1}^{s} a_{l i} \varphi(t)^{l_{i}}|\xi| u\right) d u\right|^{2} \frac{d t}{t}\right)^{\min \left\{1 / \gamma^{\prime}, 1 / 2\right\}} .
\end{aligned}
$$


Notice that

$$
\begin{aligned}
& \left(\int_{2^{k \gamma^{\prime}}}^{2^{(k+1) \gamma^{\prime}}}|h(t)|^{\gamma} \frac{d t}{t}\right)^{1 / \gamma} \\
& \quad \leq\left(\sum_{i=0}^{\left[\gamma^{\prime}\right]} \int_{2^{k \gamma^{\prime}+i}}^{2^{k \gamma^{\prime}+i+1}}|h(t)|^{\gamma} \frac{d t}{t}\right)^{1 / \gamma} \leq\left(2\left(\left[\gamma^{\prime}\right]+1\right)\|h\|_{\Delta_{\gamma}\left(\mathbb{R}_{+}\right)}^{\gamma}\right)^{1 / \gamma} \leq\left(4 \gamma^{\prime}\right)^{1 / \gamma}\|h\|_{\Delta_{\gamma}\left(\mathbb{R}_{+}\right)} .
\end{aligned}
$$

This together with (3.10) and (3.13) implies

$$
\begin{aligned}
& \left|\widehat{\sigma_{h, \Omega, k, \theta, s}}(\xi)\right| \\
& \leq\left(4 \gamma^{\prime}\right)^{\max \{1 / 2,1 / \gamma\}}\|h\|_{\Delta_{\gamma}\left(\mathbb{R}_{+}\right)} \\
& \quad \times\left(\int_{2^{k \gamma^{\prime}}}^{2^{(k+1) \gamma^{\prime}}}\left|\int_{\mathbb{R}} F_{A}(u) \exp \left(\sum_{i=1}^{s} a_{l_{i}} \varphi(t)^{l_{i}}|\xi| u\right) d u\right|^{2} \frac{d t}{t}\right)^{\min \left\{1 / \gamma^{\prime}, 1 / 2\right\}} .
\end{aligned}
$$

By some changes of variables and the properties for $\varphi$, we have

$$
\begin{aligned}
& \int_{2^{k \gamma^{\prime}}}^{2^{(k+1) \gamma^{\prime}}}\left|\int_{\mathbb{R}} F_{A}(u) \exp \left(\sum_{i=1}^{s} a_{l_{i}} \varphi(t)^{l_{i}}|\xi| u\right) d u\right|^{2} \frac{d t}{t} \\
& \leq \sum_{\mu=0}^{\left[\gamma^{\prime}\right]} \int_{2^{k \gamma^{\prime}+\mu}}^{2^{k \gamma^{\prime}+\mu+1}}\left|\int_{\mathbb{R}} F_{A}(u) \exp \left(\sum_{i=1}^{s} a_{l_{i}} \varphi(t)^{l_{i}}|\xi| u\right) d u\right|^{2} \frac{d t}{t} \\
& \leq \sum_{\mu=0}^{\left[\gamma^{\prime}\right]} \int_{\varphi\left(2^{k \gamma^{\prime}+\mu}\right)}^{\varphi\left(2^{k \gamma^{\prime}+\mu+1}\right)}\left|\int_{\mathbb{R}} F_{A}(u) \exp \left(\sum_{i=1}^{s} a_{l_{i}} t^{l}|\xi| u\right) d u\right|^{2} \frac{d t}{\varphi^{-1}(t) \varphi^{\prime}\left(\varphi^{-1}(t)\right)} \\
& \leq \frac{1}{C_{\varphi}} \sum_{\mu=0}^{\left[\gamma^{\prime}\right]} \int_{\varphi\left(2^{k \gamma^{\prime}+\mu}\right)}^{\varphi\left(2^{k \gamma^{\prime}+\mu+1}\right)}\left|\int_{\mathbb{R}} F_{A}(u) \exp \left(\sum_{i=1}^{s} a_{l_{i}} t^{l_{i}}|\xi| u\right) d u\right|^{2} \frac{d t}{t} \\
& =\frac{1}{C_{\varphi}} \sum_{\mu=0}^{\left[\gamma^{\prime}\right]} \int_{\frac{\varphi\left(2 k \gamma^{\prime}+\mu+1\right)}{\varphi\left(2^{\left.k \gamma^{\prime}+\mu\right)}\right)}}^{1}\left|\int_{\mathbb{R}} F_{A}(u) \exp \left(\sum_{i=1}^{s} a_{l_{i}} \varphi\left(2^{k \gamma^{\prime}+\mu+1}\right)^{l_{i}} t^{l_{i}}|\xi| u\right) d u\right|^{2} \frac{d t}{t} \\
& \leq \frac{1}{C_{\varphi}} \sum_{\mu=0}^{\left[\gamma^{\prime}\right]} \int_{c_{\varphi}^{-1}}^{1}\left|\int_{\mathbb{R}} F_{A}(u) \exp \left(\sum_{i=1}^{s} a_{l_{i}} \varphi\left(2^{k \gamma^{\prime}+\mu+1}\right)^{l_{i}} t^{l_{i}}|\xi| u\right) d u\right|^{2} \frac{d t}{t} \\
& \leq \frac{1}{C_{\varphi}} \sum_{\mu=0}^{\left[\gamma^{\prime}\right]} \int_{\mathbb{R}} \int_{\mathbb{R}}\left|F_{A}(u) \overline{F_{A}(v)}\right| \\
& \times\left|\int_{c_{\varphi}^{-1}}^{1} \exp \left(\sum_{i=1}^{s} a_{l_{i}} \varphi\left(2^{k \gamma^{\prime}+\mu+1}\right)^{l_{i}} t^{l_{i}}|\xi|(u-v)\right) \frac{d t}{t}\right| d u d v .
\end{aligned}
$$

Fix $\mu \in\left\{0,1, \ldots,\left[\gamma^{\prime}\right]\right\}$, we get by Lemma 2.4 that

$$
\begin{aligned}
& \left|\int_{c_{\varphi}^{-1}}^{1} \exp \left(\sum_{i=1}^{s} a_{l_{i}} \varphi\left(2^{k \gamma^{\prime}+\mu+1}\right)^{l_{i}} t^{l_{i}}|\xi|(u-v)\right) \frac{d t}{t}\right| \\
& \leq C \min \left\{1,\left(\left|a_{l_{s}} \xi\right| \varphi\left(2^{k \gamma^{\prime}+\mu+1}\right)^{l_{s}}|u-v|\right)^{-1 / l_{s}}\right\}
\end{aligned}
$$




$$
\leq C\left(\left|a_{l_{s}} \xi\right| \varphi\left(2^{k \gamma^{\prime}+i+1}\right)^{l_{i}}|u-v|\right)^{-1 /\left(l_{s} \delta\right)}
$$

where $\delta=1$ if $n \geq 3$ and $\delta=q^{\prime}$ if $n=2$. Here, $q$ is given as in the proof of Lemma 3.1. Here, the constant $C>0$ is independent of $u, v, \xi, \mu, k$, and $\left\{a_{l_{i}}\right\}_{i=1}^{s}$. In view of (3.15) with (3.16),

$$
\begin{aligned}
& \int_{2^{k \gamma^{\prime}}}^{2^{(k+1) \gamma^{\prime}}}\left|\int_{\mathbb{R}} F_{A}(u) \exp \left(\sum_{i=1}^{s} a_{l_{i}} \varphi(t)^{l_{i}}|\xi| u\right) d u\right|^{2} \frac{d t}{t} \\
& \quad \leq C \gamma^{\prime}\left(\varphi\left(2^{k \gamma^{\prime}}\right)^{l_{s}}\left|a_{l_{s}} \xi\right|\right)^{-1 /\left(l_{s} \delta\right)} \int_{\mathbb{R}} \int_{\mathbb{R}}\left|F_{A}(u) \overline{F_{A}(v)}\right||u-v|^{-1 /\left(l_{s} \delta\right)} d u d \nu .
\end{aligned}
$$

Define the function $b(u)=r\left(\xi^{\prime}\right) F_{A}\left(r\left(\xi^{\prime}\right) u+\xi_{1}^{\prime}\right)$. In view of (3.6)-(3.8) we see that $\operatorname{supp}(b) \subset$ $(-2,2)$ and $\|b\|_{L^{\infty}(\mathbb{R})} \leq C$ for $n \geq 3$ and $\|b\|_{L^{q}(\mathbb{R})} \leq C$ for $n=2$. By some changes of variables,

$$
\begin{aligned}
& \int_{\mathbb{R}} \int_{\mathbb{R}}\left|F_{A}(u) \overline{F_{A}(v)}\right||u-v|^{-1 /\left(l_{s} \delta\right)} d u d v \\
& =\left|r\left(\xi^{\prime}\right)\right|^{-1 /\left(l_{s} \delta\right)} \int_{-2}^{2} \int_{-2}^{2}|b(u) \overline{b(v)}||u-v|^{-1 /\left(l_{S} \delta\right)} d u d v .
\end{aligned}
$$

When $n \geq 3$, by the fact $\|b\|_{L^{\infty}(\mathbb{R})} \leq C$ and $\delta=1$, we get

$$
\int_{-2}^{2} \int_{-2}^{2}|b(u) \overline{b(v)}||u-v|^{-1 / l_{s}} d u d v \leq C \int_{-2}^{2} \int_{-2}^{2}|u-v|^{-1 / l_{s}} d u d v \leq C .
$$

When $n=2$, by the fact $\|b\|_{L^{q(\mathbb{R})}} \leq C$ and Hölder's inequality,

$$
\begin{aligned}
& \int_{-2}^{2} \int_{-2}^{2}|b(u) \overline{b(v)}||u-v|^{-1 /\left(l_{s} q^{\prime}\right)} d u d v \\
& \quad \leq C\|b\|_{L^{q\left(\mathbb{R}^{n}\right)}}^{2}\left(\int_{-2}^{2} \int_{-2}^{2}|u-v|^{-1 / l_{s}} d u d v\right)^{1 / q^{\prime}} \leq C .
\end{aligned}
$$

Therefore, we get from (3.18) that

$$
\int_{\mathbb{R}} \int_{\mathbb{R}}\left|F_{A}(u) \overline{F_{A}(v)}\right||u-v|^{-1 /\left(s l_{s} \delta\right)} d u d v \leq C\left|r\left(\xi^{\prime}\right)\right|^{-1 /\left(l_{s} \delta\right)} .
$$

It follows from (3.19) and (3.17) that

$$
\begin{aligned}
& \int_{2^{k \gamma^{\prime}}}^{2^{(k+1) \gamma^{\prime}}}\left|\int_{\mathbb{R}} F_{A}(u) \exp \left(\sum_{i=1}^{s} a_{l_{i}} \varphi(t)^{l_{i}}|\xi| u\right) d u\right|^{2} \frac{d t}{t} \\
& \quad \leq C \gamma^{\prime}\left(\varphi\left(2^{k \gamma^{\prime}}\right)^{l_{s}}\left|L_{s}(\xi)\right|\right)^{-1 /\left(l_{s} \delta\right)},
\end{aligned}
$$

where $C>0$ is independent of $h, \Omega, \gamma, \varrho, \xi, k$ and $\left\{a_{l_{i}}\right\}_{i=1}^{s}$. In view of (3.20) and (3.14),

$$
\left|\widehat{\sigma_{h, \Omega, k, \theta, s}}(\xi)\right| \leq C \gamma^{\prime}\|h\|_{\Delta_{\gamma}\left(\mathbb{R}_{+}\right)}\left(\varphi\left(2^{k \gamma^{\prime}}\right)^{l_{s}}\left|L_{s}(\xi)\right|\right)^{-\min \left\{1 / \gamma^{\prime}, 1 / 2\right\} /\left(l_{s} \delta\right)}
$$


where $C>0$ is independent of $h, \Omega, \gamma, \varrho, \xi, k$, and $\left\{a_{l_{i}}\right\}_{i=1}^{s}$. On the other hand, we get by (3.5), (3.10), and (3.11) that

$$
\left|\widehat{\sigma_{h, \Omega, k, \theta, s}}(\xi)\right| \leq\left\|F_{A}\right\|_{L^{1}(\mathbb{R})} \int_{2^{k \gamma^{\prime}}}^{2^{(k+1) \gamma^{\prime}}}|h(t)| \frac{d t}{t} \leq C \gamma^{\prime}\|h\|_{\Delta_{\gamma}\left(\mathbb{R}_{+}\right)}
$$

Then (3.12) follows from (3.21) and (3.22).

Lemma 3.3 Let $h \in \Delta_{\gamma}\left(\mathbb{R}_{+}\right)$for some $\gamma \in(1, \infty]$ and $\Omega$ be a $(1, \infty)$ atom satisfying $(2.1)$ (2.3) with $0<\varrho \leq 1$ and $\vartheta=\theta=(1,0, \ldots, 0) \in \mathrm{S}^{n-1}$. Let $\varphi \in \mathfrak{F}_{1}$ or $\varphi \in \mathfrak{F}_{2}$. Then, for $\gamma^{\prime}<p<$ $\infty$, there exists a constant $C>0$ independent of $h, \Omega, \gamma, \xi, \theta$, and $\left\{a_{l_{s}}\right\}_{s=1}^{\Lambda}$ such that

$$
\left\|\sup _{k \in \mathbb{Z}}|| \sigma_{h, \Omega, k, \theta, 0}|* f|\right\|_{L^{p}\left(\mathbb{R}^{n)}\right.} \leq C \gamma^{\prime}\|h\|_{\Delta_{\gamma}\left(\mathbb{R}_{+}\right)}\|f\|_{L^{p}\left(\mathbb{R}^{n}\right)}, \quad \forall f \in L^{p}\left(\mathbb{R}^{n}\right) .
$$

Proof We only consider the case $\varphi \in \mathfrak{F}_{1}$ since another one can be obtained similarly. Fix $k \in \mathbb{Z}$, by a change of variables,

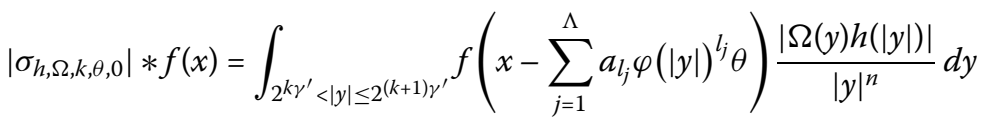

$$
\begin{aligned}
& =\int_{2^{k \gamma^{\prime}}}^{2^{(k+1) \gamma^{\prime}}} f\left(x-\sum_{j=1}^{\Lambda} a_{l_{j}} \varphi(t)^{l_{j} \theta}\right)|h(t)| \frac{d t}{t}\|\Omega\|_{L^{1}\left(S^{n-1}\right)} .
\end{aligned}
$$

It is clear that $\|\Omega\|_{L^{1}\left(S^{n-1}\right)} \leq C$. By Hölder's inequality and a change of variables, one has

$$
\begin{aligned}
& || \sigma_{h, \Omega, k, \theta, 0}|* f(x)| \\
& \quad \leq C \int_{2^{k \gamma^{\prime}}}^{2^{(k+1) \gamma^{\prime}}}\left|f\left(x-\sum_{j=1}^{\Lambda} a_{l_{j}} \varphi(t)^{l_{j} \theta}\right)\right||h(t)| \frac{d t}{t} \\
& \quad \leq C \sum_{i=0}^{\left[\gamma^{\prime}\right]} \int_{2^{k \gamma^{\prime}+i}}^{2^{k \gamma^{\prime}+i+1}}\left|f\left(x-\sum_{j=1}^{\Lambda} a_{l_{j}} \varphi(t)^{l_{j} \theta}\right)\right||h(t)| \frac{d t}{t} \\
& \quad \leq C\|h\|_{\Delta_{\gamma}\left(\mathbb{R}_{+}\right)} \sum_{i=0}^{\left[\gamma^{\prime}\right]}\left(\int_{2^{k \gamma^{\prime}+i}}^{2^{k \gamma^{\prime}+i+1}}\left|f\left(x-\sum_{j=1}^{\Lambda} a_{l_{j}} \varphi(t)^{l_{j} \theta}\right)\right|^{\gamma^{\prime}} \frac{d t}{t}\right)^{1 / \gamma^{\prime}} \\
& \quad=C\|h\|_{\Delta_{\gamma}\left(\mathbb{R}_{+}\right)} \sum_{i=0}^{\left[\gamma^{\prime}\right]}\left(\int_{\varphi\left(2^{k \gamma^{\prime}+i}\right)}^{\varphi\left(2^{k \gamma^{\prime}+i+1}\right)}\left|f\left(x-\sum_{j=1}^{\Lambda} a_{l_{j}} t^{l_{j} \theta}\right)\right|^{\gamma^{\prime}} \frac{d t}{\varphi^{-1}(t) \varphi^{\prime}\left(\varphi^{-1}(t)\right)}\right)^{1 / \gamma^{\prime}} \\
& \quad \leq C\|h\|_{\Delta_{\gamma}\left(\mathbb{R}_{+}\right)} \sum_{i=0}^{\left[\gamma^{\prime}\right]}\left(\int_{\varphi\left(2^{k \gamma^{\prime}+i}\right)}^{\varphi\left(2^{k \gamma^{\prime}+i+1}\right)}\left|f\left(x-\sum_{j=1}^{\Lambda} a_{l_{j}} t^{l_{j} \theta}\right)\right|^{\gamma^{\prime}} \frac{d t}{t}\right)^{1 / \gamma^{\prime}} \\
& \quad \leq C \gamma^{\prime}\|h\|_{\Delta_{\gamma}\left(\mathbb{R}_{+}\right)}\left(\sup _{r>0} \frac{1}{r} \int_{|t| \leq r}\left|f\left(x-\sum_{j=1}^{\Lambda} a_{l_{j}} t^{l_{j} \theta}\right)\right|^{\gamma^{\prime}} d t\right)^{1 / \gamma^{\prime}} \cdot
\end{aligned}
$$


It follows that

$$
\sup _{k \in \mathbb{Z}}|| \sigma_{h, \Omega, k, \theta, 0}|* f(x)| \leq C \gamma^{\prime}\|h\|_{\Delta_{\gamma}\left(\mathbb{R}_{+}\right)}\left(\sup _{r>0} \frac{1}{r} \int_{|t| \leq r}\left|f\left(x-\sum_{j=1}^{\Lambda} a_{l_{j}} t^{l_{j} \theta}\right)\right|^{\gamma^{\prime}} d t\right)^{1 / \gamma^{\prime}} .
$$

This together with Lemma 2.6 yields (3.23).

The following result is the main ingredient of proving Theorem 1.2.

Lemma 3.4 Let $A>0, \Lambda \in \mathbb{N} \backslash\{0\}$ and $\left\{\sigma_{k, s}: 0 \leq s \leq \Lambda\right.$ and $\left.k \in \mathbb{Z}\right\}$ be a family of uniformly bounded Borel measures on $\mathbb{R}^{n}$ with $\sigma_{k, 0}(\xi)=0$ for every $k \in \mathbb{Z}$ and $\xi \in \mathbb{R}^{n}$. For $1 \leq s \leq \Lambda$, let $\eta_{s}>1, v \geq 1, \delta_{s}, \beta_{s}>0,\left\{a_{k, s, v}\right\}$ be a sequence of positive numbers, $\ell_{s} \in \mathbb{N} \backslash\{0\}$ and $L_{s}: \mathbb{R}^{n} \rightarrow \mathbb{R}^{\ell_{s}}$ be a linear transformation. Suppose that there exists a constant $C>0$ independent of $A$ such that the following are satisfied for $k \in \mathbb{Z}, \xi \in \mathbb{R}^{n}$ and $s \in\{1, \ldots, \Lambda\}$ :

(a) $\left|\widehat{\sigma_{k, s}}(\xi)\right| \leq C A \min \left\{1,\left|a_{k, s, v} L_{s}(\xi)\right|^{-\delta_{s} / v}\right\}$;

(b) $\left.\left|\widehat{\sigma_{k, s}}(\xi)-\widehat{\sigma_{k, s-1}}(\xi) \leq C A\right| a_{k, s, v} L_{s}(\xi)\right|^{\beta_{s} / v}$;

(c) $\inf _{k \in \mathbb{Z}} \frac{a_{k+1, s, v}}{a_{k, s, v}} \geq \eta_{s}^{v}$ or $\inf _{k \in \mathbb{Z}} \frac{a_{k, s, v}}{a_{k+1, v, v}} \geq \eta_{s}^{v}$;

(d) For some $q \in(1, \infty)$, it holds that

$$
\left\|\sup _{k \in \mathbb{Z}}|| \sigma_{k, s}|* f|\right\|_{L^{q}\left(\mathbb{R}^{n}\right)} \leq C A\|f\|_{L^{q}\left(\mathbb{R}^{n)}\right)}, \quad \forall f \in L^{q}\left(\mathbb{R}^{n}\right) .
$$

Then there exists a constant $C>0$ such that

$$
\left\|\left.\sup _{k \in \mathbb{Z}}\left|\sum_{j=k}^{\infty} \sigma_{j, \Lambda} * f\right|\right|_{L^{p}\left(\mathbb{R}^{n}\right)} \leq C A\right\| f \|_{L^{p}\left(\mathbb{R}^{n)}\right.}, \quad \forall f \in L^{p}\left(\mathbb{R}^{n}\right)
$$

where $p=2$ if $q=2, p \in(q, 2]$ if $q \in(1,2)$, and $p \in\left[2, \min \left\{q, \frac{2 q}{q-1}\right\}\right)$ if $q>2$. Here, $C>0$ is independent of $A, v,\left\{L_{s}\right\}_{s=1}^{\Lambda}, f$, but may depend on $p, n, \Lambda,\left\{\ell_{s}\right\}_{s=1}^{\Lambda},\left\{\beta_{s}\right\}_{s=1}^{\Lambda}$ and $\left\{\delta_{s}\right\}_{s=1}^{\Lambda},\left\{\eta_{s}\right\}_{s=1}^{\Lambda}$.

Proof We shall adopt the method following from [22] to prove this lemma. For simplicity, we only consider the case $\inf _{k \in \mathbb{Z}} \frac{a_{k+1, s, v}}{a_{k, s, v}} \geq \eta_{s}^{v}$, since another one can be proved similarly. For $s \in\{1, \ldots, \Lambda\}$, we set $r_{s}=\operatorname{rank}\left(L_{s}\right)$ and let $\pi_{r_{s}}^{n}(\xi)=\left(\xi_{1}, \ldots, \xi_{r_{s}}\right)$ be the projection from $\mathbb{R}^{n}$ to $\mathbb{R}^{r_{s}}$. Invoking [22, Lemma 6.1], there exist two nonsingular linear transformations $H_{s}: \mathbb{R}^{r_{s}} \rightarrow \mathbb{R}^{r_{s}}$ and $G_{s}: \mathbb{R}^{n} \rightarrow \mathbb{R}^{n}$ such that

$$
\left|H_{s} \pi_{r_{s}}^{n} G_{s}(\xi)\right| \leq\left|L_{s}(\xi)\right| \leq \ell_{s}\left|H_{s} \pi_{r_{s}}^{n} G_{s}(\xi)\right|
$$

Let $\phi \in \mathcal{C}_{0}^{\infty}(\mathbb{R})$ be such that $\operatorname{supp}(\phi)=\{|t| \leq 1\}$ and $\phi(t) \equiv 1$ for $|t|<1 / 2$. For $s \in\{1, \ldots, \Lambda\}$, we define a sequence of measures $\left\{\mu_{k, s}\right\}_{k \in \mathbb{Z}}$ on $\mathbb{R}^{n}$ by

$$
\widehat{\mu_{k, s}}(\xi)=\widehat{\sigma_{k, s}}(\xi) \prod_{j=s+1}^{\Lambda} \phi\left(\left|a_{k, j, v} H_{j} \pi_{r_{j}}^{n} G_{j}(\xi)\right|\right)-\widehat{\sigma_{k, s-1}}(\xi) \prod_{j=s}^{\Lambda} \phi\left(\left|a_{k, j, v} H_{j} \pi_{r_{j}}^{n} G_{j}(\xi)\right|\right) .
$$

It is not difficult to see that

$$
\sigma_{k, \Lambda}=\sum_{s=1}^{\Lambda} \mu_{k, s}
$$


In view of (3.27) we write

$$
\sup _{k \in \mathbb{Z}}\left|\sum_{j=k}^{\infty} \sigma_{j, \Lambda} * f\right| \leq \sum_{s=1}^{\Lambda} \sup _{k \in \mathbb{Z}}\left|\sum_{j=k}^{\infty} \mu_{j, s} * f\right|=: \sum_{s=1}^{\Lambda} T_{s}^{*}(f) .
$$

Therefore, for (3.24), it suffices to show that

$$
\left\|T_{s}^{*}(f)\right\|_{L^{p}\left(\mathbb{R}^{n)}\right.} \leq C_{p} A\|f\|_{L^{p}\left(\mathbb{R}^{n}\right)}
$$

for all $1 \leq s \leq \Lambda$, where $p=2$ if $q=2$, and $p \in(q, 2]$ if $q \in(1,2)$, and $p \in\left[2, \min \left\{q, \frac{2 q}{q-1}\right\}\right)$ if $q>2$. Here, $C_{p}>0$ is independent of $A, v, L_{s}, f$, but may depend on $p, n, \Lambda, \ell_{s}, \beta_{s}, \delta_{s}, \eta_{s}$.

Let $\psi \in \mathcal{S}(\mathbb{R})$ be such that $\psi(\xi) \equiv 1$ when $|\xi|<1$ and $\psi(\xi) \equiv 0$ when $|\xi|>\eta_{s}$. Define the function $\Phi_{k}$ by $\widehat{\Phi_{k}}(\xi)=\psi\left(a_{k, s, v}\left|H_{s} \pi_{r_{s}}^{n} G_{s}(\xi)\right|\right)$. Write

$$
\begin{aligned}
\sum_{j=k}^{\infty} \mu_{j, s} * f & =\Phi_{k} * T_{s}(f)+\left(\delta-\Phi_{k}\right) * \sum_{j=k}^{\infty} \mu_{j, s} * f-\Phi_{k} * \sum_{j=-\infty}^{k-1} \mu_{j, s} * f \\
& =: I_{k, 1}(f)+I_{k, 2}(f)+I_{k, 3}(f),
\end{aligned}
$$

where $\delta$ is the Dirac delta function and

$$
T_{s}(f)=\sum_{k \in \mathbb{Z}} \mu_{k, s} * f
$$

It follows that

$$
T_{s}^{*}(f) \leq \sup _{k \in \mathbb{Z}}\left|I_{k, 1}(f)\right|+\sup _{k \in \mathbb{Z}}\left|I_{k, 2}(f)\right|+\sup _{k \in \mathbb{Z}}\left|I_{k, 3}(f)\right|
$$

We first prove that

$$
\left\|T_{s}(f)\right\|_{L^{p}\left(\mathbb{R}^{n}\right)} \leq C_{p} A\|f\|_{L^{p}\left(\mathbb{R}^{n}\right)}
$$

for $p \in\left(\frac{2 q}{q+1}, \frac{2 q}{q-1}\right)$ and $s \in\{1, \ldots, \Lambda\}$, where $C_{p}>0$ is independent of $A, v, L_{s}, f$, but may depend on $p, n, \Lambda, \ell_{s}, \beta_{s}, \delta_{s}, \eta_{s}$. In view of assumptions (a) and (b) and (3.26),

$$
\begin{aligned}
& \left|\widehat{\mu_{k, s}}(\xi)\right| \leq C A\left(\left|a_{k, s, v} L_{s}(\xi)\right|^{\beta_{s} / v}+\left|a_{k, s, v} L_{s}(\xi)\right|^{1 / v}\right) \\
& \left|\widehat{\mu_{k, s}}(\xi)\right| \leq C A \min \left\{1,\left|a_{k, s, v} L_{s}(\xi)\right|^{-\delta_{s} / v}+\left|a_{k, s, v} L_{s}(\xi)\right|^{-1 / v}\right\} .
\end{aligned}
$$

Let $\left\{\Psi_{k, s}\right\}_{k \in \mathbb{Z}}$ be a sequence of nonnegative functions in $\mathcal{C}_{0}^{\infty}(\mathbb{R})$ such that

$$
\begin{aligned}
& \operatorname{supp}\left(\Psi_{k, s}\right) \subset\left[a_{k+1, s, v}^{-1}, a_{k-1, s, v}^{-1}\right], \quad \sum_{k \in \mathbb{Z}} \Psi_{k, s}^{2}(t)=1, \\
& \left|\left(\frac{d}{d t}\right)^{j} \Psi_{k, s}(t)\right| \leq C_{j}|t|^{-j}(j=1,2, \ldots) \quad \text { for all } t>0 \text { and } j \in \mathbb{N},
\end{aligned}
$$

where $C_{j}$ are independent of $s, v$, and $k$. Define the Fourier multiplier operator $S_{j, s}$ by

$$
\widehat{S_{j, s} f}(\xi)=\Psi_{j, s}\left(\left|H_{s} \pi_{r_{s}}^{n} G_{s}(\xi)\right|\right) \hat{f}(\xi) \quad \text { for } j \in \mathbb{Z}
$$


Thus, the operator $T_{s}$ can be decomposed as

$$
T_{s}(f)=\sum_{k \in \mathbb{Z}} \mu_{k, s} * \sum_{j \in \mathbb{Z}} S_{j+k, s} S_{j+k, s} f=\sum_{j \in \mathbb{Z}} \sum_{k \in \mathbb{Z}} S_{j+k, s}\left(\mu_{k, s} * S_{j+k, s} f\right)=: \sum_{j \in \mathbb{Z}} T_{s, j}(f) .
$$

By the Littlewood-Paley theory, Plancherel's theorem, and assumption (c), we then use (3.33) and (3.34) to obtain

$$
\begin{aligned}
\left\|T_{s, j}(f)\right\|_{L^{2}\left(\mathbb{R}^{n}\right)} & \leq C\left\|\left(\sum_{k \in \mathbb{Z}}\left|\mu_{k, s} * S_{j+k, s} f\right|^{2}\right)^{1 / 2}\right\|_{L^{2}\left(\mathbb{R}^{n}\right)} \\
& \leq C\left(\sum_{k \in \mathbb{Z}} \int_{a_{j+k+1, s, v}^{-1} \leq \mid H_{s} \pi_{r_{s}}^{n} G_{s}(\xi) \leq a_{j+k-1, s, v}^{-1}}\left|\widehat{\mu_{k, s}}(\xi)\right|^{2}|\hat{f}(\xi)|^{2} d \xi\right)^{1 / 2} \\
& \leq C A \eta_{s}^{-c|j|}\|f\|_{L^{2}\left(\mathbb{R}^{n}\right)}
\end{aligned}
$$

for some $c>0$, where $C>0$ is independent of $A, v, L_{s}, f$, but may depend on $\ell_{s}, \beta_{s}, \delta_{s}, \eta_{s}$.

On the other hand, by our assumption (d), (3.26) and a well-known result on maximal functions (see [22]), there exists a constant $C>0$ independent of $A, v, L_{s}$ such that

$$
\left\|\sup _{k \in \mathbb{Z}}|| \mu_{k, s}|* f|\right\|_{L^{q}\left(\mathbb{R}^{n}\right)} \leq C A\|f\|_{L^{q}\left(\mathbb{R}^{n)}\right.}, \quad \forall f \in L^{q}\left(\mathbb{R}^{n}\right)
$$

for any $1 \leq s \leq \Lambda$. Using (3.38) and the lemma in [16, pp. 544],

$$
\left\|\left(\sum_{k \in \mathbb{Z}}\left|\mu_{k, s} * g_{k}\right|^{2}\right)^{1 / 2}\right\|_{L^{p}\left(\mathbb{R}^{n}\right)} \leq C_{p} A\left\|\left(\sum_{k \in \mathbb{Z}}\left|g_{k}\right|^{2}\right)^{1 / 2}\right\|_{L^{p}\left(\mathbb{R}^{n}\right)}
$$

for $|1 / p-1 / 2|=1 /(2 q)$ and arbitrary functions $\left\{g_{k}\right\}_{k} \in L^{p}\left(\ell^{2}, \mathbb{R}^{n}\right)$. Here, $C_{p}>0$ is independent of $A, v, L_{s}$. Combining (3.39) with the Littlewood-Paley theory implies

$$
\begin{aligned}
\left\|T_{s, j}(f)\right\|_{L^{p}\left(\mathbb{R}^{n)}\right.} & \leq C\left\|\left(\sum_{k \in \mathbb{Z}}\left|\mu_{k, s} * S_{j+k, f}\right|^{2}\right)^{1 / 2}\right\|_{L^{p}\left(\mathbb{R}^{n}\right)} \\
& \leq C A\left\|\left(\sum_{k \in \mathbb{Z}}\left|S_{j+k, s} f\right|^{2}\right)^{1 / 2}\right\|_{L^{p}\left(\mathbb{R}^{n)}\right.} \leq C A\|f\|_{L^{p}\left(\mathbb{R}^{n)}\right.},
\end{aligned}
$$

where $|1 / p-1 / 2|=1 /(2 q)$ and $C>0$ is independent of $A, v, L_{s}$. By interpolation between (3.37) and (3.40), we have that, for any $p \in\left(\frac{2 q}{q+1}, \frac{2 q}{q-1}\right)$ and some $c^{\prime}>0$,

$$
\left\|T_{s, j}(f)\right\|_{L^{p}\left(\mathbb{R}^{n)}\right.} \leq C A \eta_{s}^{-c^{\prime}|j|}\|f\|_{L^{p}\left(\mathbb{R}^{n}\right)}
$$

Inequality (3.41) together with (3.36) and Minkowski's inequality implies (3.32).

By (3.32) and a well-known result on maximal functions (see [22]), we have that, for all $p \in\left(\frac{2 q}{q+1}, \frac{2 q}{q-1}\right)$,

$$
\left\|\sup _{k \in \mathbb{Z}}\left|I_{k, 1}(f)\right|\right\|_{L^{p}\left(\mathbb{R}^{n)}\right.} \leq C\left\|T_{S}(f)\right\|_{L^{p}\left(\mathbb{R}^{n}\right)} \leq C_{p} A\|f\|_{L^{p}\left(\mathbb{R}^{n}\right)},
$$

where $C_{p}>0$ is independent of $A, v, L_{s}, f$, but may depend on $p, n, \ell_{s}, \beta_{s}, \delta_{s}, \eta_{s}$. 
We now estimate $\left\|\sup _{k \in \mathbb{Z}}\left|I_{k, 2}(f)\right|\right\|_{L^{p}\left(\mathbb{R}^{n}\right)}$. Write

$$
\sup _{k \in \mathbb{Z}}\left|I_{k, 2}(f)\right| \leq \sum_{j=0}^{\infty} \sup _{k \in \mathbb{Z}}\left|\left(\delta-\Phi_{k}\right) * \mu_{j+k, s} * f\right|=: \sum_{j=0}^{\infty} I_{j}(f) .
$$

An application of (3.38) shows that

$$
\left\|I_{j}(f)\right\|_{L^{q}\left(\mathbb{R}^{n}\right)} \leq C\left\|\sup _{k \in \mathbb{Z}}|| \mu_{j+k, s}|*| f||\right\|_{L^{q}\left(\mathbb{R}^{n}\right)} \leq C A\|f\|_{L^{q}\left(\mathbb{R}^{n}\right)} .
$$

In view of Plancherel's theorem, (3.25), and (3.33), we have that, for some $c>0$,

$$
\begin{aligned}
\left\|I_{j}(f)\right\|_{L^{2}\left(\mathbb{R}^{n}\right)}^{2} & \leq\left\|\left(\sum_{k \in \mathbb{Z}}\left|\left(\delta-\Phi_{k}\right) * \mu_{j+k, s} * f\right|^{2}\right)^{1 / 2}\right\|_{L^{2}\left(\mathbb{R}^{n}\right)}^{2} \\
& \leq \sum_{k \in \mathbb{Z}} \int_{\left\{a_{k, s, v}\left|H_{s} \pi_{r s}^{n} G_{s}(\xi)\right| \geq 1\right\}}\left|\widehat{\mu_{j+k, s}}(\xi)\right|^{2}|\hat{f}(\xi)|^{2} d \xi \\
& \leq \sum_{k \in \mathbb{Z}} \sum_{i=-\infty}^{k} \int_{\left\{a_{i, s, v}^{-1} \leq\left|L_{s}(\xi)\right|<a_{i-1, s, v}^{-1}\right\}}\left|\widehat{\mu_{j+k, s}}(\xi)\right|^{2}|\hat{f}(\xi)|^{2} d \xi \\
& \leq C \sum_{k \in \mathbb{Z}} \sum_{i=-\infty}^{k} A^{2} \eta_{s}^{-c(j+k-i)} \int_{\left\{a_{i, s, v}^{-1} \leq L_{s}(\xi) \mid<a_{i-1, s, v}^{-1}\right\}}|\hat{f}(\xi)|^{2} d \xi \\
& \leq C A^{2} \eta_{s}^{-j c} \sum_{i=0}^{\infty} \eta_{s}^{-i c}\|f\|_{L^{2}\left(\mathbb{R}^{n}\right)}^{2} \\
& \leq C A^{2} \eta_{s}^{-j c}\|f\|_{L^{2}\left(\mathbb{R}^{n}\right)}^{2} .
\end{aligned}
$$

It follows that

$$
\left\|I_{j}(f)\right\|_{L^{2}\left(\mathbb{R}^{n}\right)} \leq C A \eta_{s}^{-j c / 2}\|f\|_{L^{2}\left(\mathbb{R}^{n}\right)} .
$$

An interpolation between (3.44) and (3.45) gives that

$$
\left\|I_{j}(f)\right\|_{L^{p}\left(\mathbb{R}^{n)}\right.} \leq C A \eta_{s}^{-\tau j}\|f\|_{L^{p}\left(\mathbb{R}^{n}\right)}
$$

for some $\tau>0$ and $p \in[2, q]$ if $q>2$ or $p \in(q, 2]$ if $q \in(1,2)$ or $p=2$ if $q=2$. Combining this with (3.43) leads to

$$
\left\|\sup _{k \in \mathbb{Z}}\left|I_{k, 2}(f)\right|\right\|_{L^{p}\left(\mathbb{R}^{n}\right)} \leq C A\|f\|_{L^{p}\left(\mathbb{R}^{n}\right)}
$$

for $p \in[2, q]$ if $q>2$ or $p \in(q, 2]$ if $q \in(1,2)$ or $p=2$ if $q=2$.

It remains to estimate $\left\|\sup _{k \in \mathbb{Z}}\left|I_{k, 3}(f)\right|\right\|_{L^{p}\left(\mathbb{R}^{n}\right)}$. Write

$$
\sup _{k \in \mathbb{Z}}\left|I_{k, 3}(f)\right|=\sup _{k \in \mathbb{Z}}\left|\sum_{j=1}^{\infty} \Phi_{k} * \mu_{k-j, s} * f\right| \leq \sum_{j=1}^{\infty} \sup _{k \in \mathbb{Z}}\left|\Phi_{k} * \mu_{k-j, s} * f\right|=: \sum_{j=1}^{\infty} J_{j}(f) .
$$


In view of (3.38), one can get

$$
\left\|J_{j}(f)\right\|_{L^{q\left(\mathbb{R}^{n}\right)}} \leq C\left\|\sup _{k \in \mathbb{Z}}|| \mu_{j-k, s}|*| f||\right\|_{L^{q\left(\mathbb{R}^{n}\right)}} \leq C A\|f\|_{L^{q}\left(\mathbb{R}^{n}\right)}
$$

In view of Plancherel's theorem, we use (3.33) and (3.25) to get

$$
\begin{aligned}
\| J_{j}(f) & \|_{L^{2}\left(\mathbb{R}^{n}\right)} \\
\leq & \left\|\left(\sum_{k \in \mathbb{Z}}\left|\Phi_{k} * \mu_{k-j, s} * f\right|^{2}\right)^{1 / 2}\right\|_{L^{2}\left(\mathbb{R}^{n}\right)} \\
\leq & \left(\sum_{k \in \mathbb{Z}} \int_{\left\{a_{k, s, v}\left|H_{s} \pi_{r_{s}}^{n} G_{s}(\xi)\right| \leq \eta_{s}\right\}}\left|\widehat{\mu_{k-j, s}}(\xi)\right|^{2}|\hat{f}(\xi)|^{2} d \xi\right)^{1 / 2} \\
\leq & C\left(\int_{\mathbb{R}^{n}} \sum_{k \in \mathbb{Z}}\left|\widehat{\mu_{k-j, s}}(\xi)\right|^{2} \chi_{\left\{a_{k, s, v}\left|L_{s}(\xi)\right| \leq \ell_{s} \eta_{s}\right\}}|\hat{f}(\xi)|^{2} d \xi\right)^{1 / 2} \\
\leq & C A\left(\eta_{s}^{-\beta_{s} j}+\eta_{s}^{-j}\right)\|f\|_{L^{2}\left(\mathbb{R}^{n}\right)} \\
& \times\left(\sup _{\xi \in \mathbb{R}^{n}} \sum_{k \in \mathbb{Z}}\left(\left|a_{k, s, v} L_{s}(\xi)\right|^{2 \beta_{s} / v}+\left|a_{k, s, v} L_{s}(\xi)\right|^{2 / v}\right) \chi_{\left\{a_{k, s, v}\left|L_{s}(\xi)\right| \leq \ell_{s} \eta_{s}\right\}}\right)^{1 / 2} \\
\leq & C A\left(\eta_{s}^{-\beta_{s} j}+\eta_{s}^{-j}\right)\|f\|_{L^{2}\left(\mathbb{R}^{n}\right)},
\end{aligned}
$$

where in the last inequality of (3.49) we have used the properties of lacunary sequence and the fact that $\ell_{s} \eta_{s}>1, v \geq 1$. Here, $C>0$ is independent of $A, v, L_{s}$, but may depend on $n, \ell_{s}, \beta_{s}, \delta_{s}, \eta_{s}$. An interpolation between (3.48) and (3.49) leads to

$$
\left\|J_{j}(f)\right\|_{L^{p}\left(\mathbb{R}^{n}\right)} \leq C A\left(\eta_{s}^{-\theta \beta_{s} j}+\eta_{s}^{-\theta j}\right)\|f\|_{L^{2}\left(\mathbb{R}^{n}\right)}
$$

for some $\theta>0$, where $p \in[2, q]$ if $q>2$ or $p \in(q, 2]$ if $q \in(1,2)$ or $p=2$ if $q=2$. By (3.47), (3.50), and Minkowski's inequality,

$$
\left\|\sup _{k \in \mathbb{Z}}\left|I_{k, 3}(f)\right|\right\|_{L^{p}\left(\mathbb{R}^{n}\right)} \leq C A\|f\|_{L^{p}\left(\mathbb{R}^{n}\right)}
$$

for $p \in[2, q]$ if $q>2$ or $p \in(q, 2]$ if $q \in(1,2)$ or $p=2$ if $q=2$. Then (3.29) follows from (3.31), (3.42), (3.46), and (3.51). This finishes the proof of Lemma 3.4.

The following result is the main ingredient of proving Theorem 1.1.

Lemma 3.5 Let $\Lambda, v \in \mathbb{N} \backslash\{0\}$. For $1 \leq s \leq \Lambda$, let $\left\{a_{k, s, v}\right\}_{k \in \mathbb{Z}}$ be a lacunary sequence of positive numbers. For $1 \leq s \leq \Lambda$, let $\delta_{s}>0, \eta_{s}>1, \ell_{s} \in \mathbb{N} \backslash\{0\}$, and $L_{s}: \mathbb{R}^{n} \rightarrow \mathbb{R}^{\ell_{s}}$ be linear transformations. Let $\left\{\sigma_{s, k}: 0 \leq s \leq \Lambda\right.$ and $\left.k \in \mathbb{Z}\right\}$ be a family of measures on $\mathbb{R}^{n}$ with $\sigma_{0, k}=0$ for every $k \in \mathbb{Z}$. Suppose that there exist $p_{0}, q_{0}>1$ satisfying $\left(p_{0}, q_{0}\right) \neq(2,2)$ and $c, A>0$ independent of $v$ and $\left\{L_{s}\right\}_{s=1}^{\Lambda}$ such that the following conditions are satisfied for any $1 \leq s \leq \Lambda$, $k \in \mathbb{Z}, \xi \in \mathbb{R}^{n}$, and $\left\{g_{k, j}\right\} \in L^{p_{0}}\left(\mathbb{R}^{n}, \ell^{q_{0}}\left(\ell^{2}\right)\right)$ :

(a) $\left|\widehat{\sigma_{s, k}}(\xi)\right| \leq c A \min \left\{1,\left|a_{k, s, v} L_{s}(\xi)\right|^{-\delta_{s} / v}\right\}$;

(b) $\left|\widehat{\sigma_{s, k}}(\xi)-\widehat{\sigma_{s-1, k}}(\xi)\right| \leq c A\left|a_{k, s, v} L_{s}(\xi)\right|^{1 / v}$;

(c) $\inf _{k \in \mathbb{Z}} \frac{a_{k+1, s, v}}{a_{k, s, v}} \geq \eta_{s}^{v}$ or $\inf _{k \in \mathbb{Z}} \frac{a_{k, s, v}}{a_{k+1, s, v}} \geq \eta_{s}^{v}$; 
(d)

$$
\begin{aligned}
& \left\|\left(\sum_{j \in \mathbb{Z}}\left(\sum_{k \in \mathbb{Z}}\left|\sigma_{s, k} * g_{k, j}\right|^{2}\right)^{q_{0} / 2}\right)^{1 / q_{0}}\right\|_{L^{p_{0}\left(\mathbb{R}^{n}\right)}} \\
& \quad \leq c A\left\|\left(\sum_{j \in \mathbb{Z}}\left(\sum_{k \in \mathbb{Z}}\left|g_{k, j}\right|^{2}\right)^{q_{0} / 2}\right)^{1 / q_{0}}\right\|_{L^{p_{0}\left(\mathbb{R}^{n}\right)}} .
\end{aligned}
$$

Then, for $\alpha \in \mathbb{R}$ and $(1 / p, 1 / q) \in B_{1} B_{2} \backslash\left\{\left(1 / p_{0}, 1 / q_{0}\right),(1 / 2,1 / 2)\right\}$, there exists a constant $C>0$ independent of $v$ and $\left\{L_{s}\right\}_{s=1}^{\Lambda}$ such that

$$
\left\|\sum_{k \in \mathbb{Z}} \sigma_{\Lambda, k} * f\right\|_{\dot{F}_{\alpha}^{p, q}\left(\mathbb{R}^{n}\right)} \leq C A\|f\|_{\dot{F}_{\alpha}^{p, q}\left(\mathbb{R}^{n}\right)},
$$

where $B_{1}=(1 / 2,1 / 2), B_{2}=\left(1 / p_{0}, 1 / q_{0}\right)$ and $B_{1} B_{2}$ is the line segment from $B_{1}$ to $B_{2}$.

Proof Assume that $\inf _{k \in \mathbb{Z}} \frac{a_{k+1, s, v}}{a_{k, s, v}} \geq \eta_{s}^{v}$ for all $1 \leq s \leq \Lambda$, the corresponding result has been proved in [27, Lemma 2.5]. Similar arguments will give the corresponding result for the case $\inf _{k \in \mathbb{Z}} \frac{a_{k, s, v}}{a_{k+1, s, v}} \geq \eta_{s}^{v}$. The details are omitted.

In order to prove Theorem 1.3, we need the following characterization of the TriebelLizorkin spaces.

Lemma $3.6([41])$ Let $0<\alpha<1,1<p<\infty, 1<q \leq \infty$, and $1 \leq r<\min \{p, q\}$. Then

$$
\|f\|_{\dot{F}_{\alpha}^{p, q}\left(\mathbb{R}^{n}\right)} \approx\left\|\left(\sum_{k \in \mathbb{Z}} 2^{k q \alpha}\left(\int_{\Re_{n}}\left|\triangle_{2^{-k} \zeta}(f)\right|^{r} d \zeta\right)^{q / r}\right)^{1 / q}\right\|_{L^{p}\left(\mathbb{R}^{n}\right)} .
$$

Our main ingredient of proving Theorem 1.3 is the following boundedness criterion.

Lemma 3.7 Let $v \geq 1, \Lambda \in \mathbb{N} \backslash\{0\}$, and $\left\{\sigma_{k, s}: k \in \mathbb{Z}, 1 \leq s \leq \Lambda\right\}$ be a family of Borel measures on $\mathbb{R}^{n}$ with $\sigma_{k, 0}=0$ for all $k \in \mathbb{Z}$. Let $\left|\sigma_{k, s}\right|$ be the total variation of $\sigma_{k, s}$. Let $\left\{a_{k, s, v}\right\}_{k \in \mathbb{Z}}$ be a lacunary sequence of positive numbers. For $1 \leq s \leq \Lambda$, let $\eta_{s}>1, \beta_{s}, \gamma_{s}>0, M_{s} \in \mathbb{N} \backslash\{0\}$, and $L_{s}: \mathbb{R}^{n} \rightarrow \mathbb{R}^{M_{s}}$ be linear transformations. Suppose that there exist $C, A>0$ independent of $v$ such that, for $1 \leq s \leq \Lambda, k \in \mathbb{Z}$, and $\xi \in \mathbb{R}^{n}$, the following conditions are satisfied:

(a) $\max \left\{\left|\widehat{\sigma_{k, s}}(\xi)-\widehat{\sigma_{k, s-1}}(\xi)\right|,|| \widehat{\sigma_{k, s}}\left|(\xi)-\widehat{\left|\sigma_{k, s-1}\right|}(\xi)\right|\right\} \leq C A\left|a_{k, s, v} L_{s}(\xi)\right|^{1 / v}$;

(b) $\max \left\{\left|\widehat{\sigma_{k, s}}(\xi)\right|,\left|\widehat{\sigma_{k, s}}\right|(\xi) \mid\right\} \leq C A \min \left\{1,\left|a_{k, s, v} L_{s}(\xi)\right|^{-\beta_{s} / v}\right\}$;

(c) There exists $\vartheta \in \mathbb{R}^{n}$ such that $\sup _{k \in \mathbb{Z}}|| \sigma_{k, 0}|* f(x)| \leq C A|f(x+\vartheta)|$ for any $x \in \mathbb{R}^{n}$;

(d) $\inf _{k \in \mathbb{Z}} \frac{a_{k+1, s, v}}{a_{k, s, v}} \geq \eta_{s}^{v}$ or $\inf _{k \in \mathbb{Z}} \frac{a_{k, s, v}}{a_{k+1, s, v}} \geq \eta_{s}^{v}$;

(e) There exist $p_{0}, q_{0}>1$ satisfying $\left(p_{0}, q_{0}\right) \neq(2,2), 1<r_{0}<\min \left\{p_{0}, q_{0}\right\}$, and $2 \leq u<\infty$ such that

$$
\begin{aligned}
& \left\|\left(\sum_{l \in \mathbb{Z}}\left\|\left(\sum_{k \in \mathbb{Z}}|| \sigma_{k, s}\left|* g_{l, \zeta, k}\right|^{u}\right)^{1 / u}\right\|_{L^{r_{0}\left(\Re_{n}\right)}}^{q_{0}}\right)^{1 / q_{0}}\right\|_{L^{p_{0}\left(\mathbb{R}^{n}\right)}} \\
& \quad \leq C A\left\|\left(\sum_{l \in \mathbb{Z}}\left\|\left(\sum_{k \in \mathbb{Z}}\left|g_{l, \zeta, k}\right|^{u}\right)^{1 / u}\right\|_{L^{r_{0}\left(\Re_{n}\right)}}^{q_{0}}\right)^{1 / q_{0}}\right\|_{L^{p_{0}\left(\mathbb{R}^{n}\right)}} .
\end{aligned}
$$


Then, for $\alpha \in(0,1)$ and $(1 / p, 1 / q) \in P_{1} P_{2} \backslash\left\{\left(1 / p_{0}, 1 / q_{0}\right)\right\}$, there exists a constant $C>0$ independent of $A$ and $v$ such that

$$
\begin{aligned}
& \left\|\left(\sum_{l \in \mathbb{Z}} 2^{l q \alpha}\left(\int_{\Re_{n}} \sup _{k \in \mathbb{Z}}|| \sigma_{k, s}|*| \triangle_{2^{-l}} f|| d \zeta\right)^{q}\right)^{1 / q}\right\|_{L^{p}\left(\mathbb{R}^{n)}\right.} \leq C A\|f\|_{\dot{F}_{\alpha}^{p, q}\left(\mathbb{R}^{n)}\right.}, \\
& \quad \forall 1 \leq s \leq \Lambda
\end{aligned}
$$

Here, $P_{1} P_{2}$ denotes the line segment from $P_{1}$ to $P_{2}$ with $P_{1}=(1 / 2,1 / 2)$ and $P_{2}=\left(1 / p_{0}, 1 / q_{0}\right)$.

(g) Suppose also that the following inequality holds for $1 \leq s \leq \Lambda$ :

$$
\begin{aligned}
& \left\|\left(\sum_{j \in \mathbb{Z}}\left(\sum_{k \in \mathbb{Z}}\left|\sigma_{k, s} * g_{k, j}\right|^{2}\right)^{q_{0} / 2}\right)^{1 / q_{0}}\right\|_{L^{p_{0}\left(\mathbb{R}^{n}\right)}} \\
& \leq C A\left\|\left(\sum_{j \in \mathbb{Z}}\left(\sum_{k \in \mathbb{Z}}\left|g_{k, j}\right|^{2}\right)^{q_{0} / 2}\right)^{1 / q_{0}}\right\|_{L^{p_{0}\left(\mathbb{R}^{n}\right)}} .
\end{aligned}
$$

Then, for $\alpha \in(0,1)$ and $(1 / p, 1 / q) \in P_{1} P_{2} \backslash\left\{\left(1 / p_{0}, 1 / q_{0}\right),(1 / 2,1 / 2)\right\}$, there exists a constant $C>0$ independent of $A$ and $v$ such that

$$
\left\|\left(\sum_{l \in \mathbb{Z}} 2^{l q \alpha}\left(\int_{\mathfrak{R}_{n}} \sup _{k \in \mathbb{Z}}\left|\sum_{j=k}^{\infty} \sigma_{j, \Lambda} * \Delta_{2^{-l_{\zeta}}} f\right| d \zeta\right)^{q}\right)^{1 / q}\right\|_{L^{p}\left(\mathbb{R}^{n}\right)} \leq C A\|f\|_{\dot{F}_{\alpha}^{p, q}\left(\mathbb{R}^{n}\right)} .
$$

Proof The lemma can be proved by the arguments similar to those used in deriving [30, Lemma 2.9]. We omit the details.

\subsection{Proofs of Theorems 1.1-1.3}

Proof of Theorem 1.1 Let $h, \Omega, P, \varphi$ be given as in Theorem 1.1. Invoking Lemma 2.1, there exist a sequence of complex numbers $\left\{c_{j}\right\}_{j=1}^{\infty}$ and a sequence of $(1, \infty)$ atoms $\left\{\Omega_{j}\right\}_{j=1}^{\infty}$ such that $\Omega=\sum_{j=1}^{\infty} c_{j} \Omega_{j}$ and $\|\Omega\|_{H^{1}\left(S^{n-1}\right)} \approx \sum_{j=1}^{\infty}\left|c_{j}\right|$. By the definition of $T_{h, \Omega, P, \varphi}$, one has

$$
T_{h, \Omega, P, \varphi} f=\sum_{j=1}^{\infty} c_{j} T_{h, \Omega_{j}, P, \varphi} f
$$

In view of (3.52) and the definition of $\dot{F}_{\alpha}^{p, q}\left(\mathbb{R}^{n}\right)$, we have that, for $1<p, q<\infty$ and $\alpha \in \mathbb{R}$,

$$
\left\|T_{h, \Omega, P, \varphi} f\right\|_{\dot{F}_{\alpha}^{p, q}\left(\mathbb{R}^{n)}\right.} \leq \sum_{j=1}^{\infty}\left|c_{j}\right|\left\|T_{h, \Omega, P, P, \varphi} f\right\|_{\dot{F}_{\alpha}^{p, q}\left(\mathbb{R}^{n}\right)}
$$

Therefore, to prove Theorem 1.1, it suffices to prove that there exists $C>0$ is independent of $h, \gamma, \Omega$ and the coefficients of $P$ such that

$$
\left\|T_{h, \Omega, P, \varphi} f\right\|_{\dot{F}_{\alpha}^{p, q}\left(\mathbb{R}^{n}\right)} \leq C \gamma^{\prime}\|h\|_{\Delta_{\gamma}\left(\mathbb{R}_{+}\right)}\|f\|_{\dot{F}_{\alpha}^{p, q}\left(\mathbb{R}^{n}\right)},
$$

holds for any $(1, \infty)$ atom $\Omega$ and $\alpha \in \mathbb{R}$ and $(p, q) \in \mathcal{R}_{\gamma}$. 
Given a $(1, \infty)$ atom $\Omega$ satisfying (2.6)-(2.8) with $0<\varrho \leq 1$ and $\vartheta \in S^{n-1}$. Without loss of generality we may assume that $\vartheta=\theta=(1,0, \ldots, 0)$. By the definition of $\sigma_{h, \Omega, k, \theta, \Lambda}$, we have

$$
T_{h, \Omega, P, \varphi} f=\sum_{k \in \mathbb{Z}} \sigma_{h, \Omega, k, \theta, \Lambda} * f .
$$

Note that if $\varphi \in \mathfrak{F}_{1}$ or $\varphi \in \mathfrak{F}_{2}$, there exist $C_{1}, C_{2}>0$ depending only on $\varphi$ such that

$$
C_{1} \leq \frac{\varphi(2 t)}{\varphi(t)} \leq C_{2}, \quad \forall t>0
$$

In view of (3.55) and Lemma 3.1,

$$
\left|\widehat{\sigma_{h, \Omega, k, \theta, s}}(\xi)-\widehat{\sigma_{h, \Omega, k, \theta, s-1}}(\xi)\right| \leq C \gamma^{\prime}\|h\|_{\Delta_{\gamma}\left(\mathbb{R}_{+}\right)} \min \left\{1,\left(\varphi\left(2^{k \gamma^{\prime}}\right)^{l_{s}}\left|L_{s}(\xi)\right|\right)^{1 / \gamma^{\prime}}\right\} .
$$

By the properties of $\varphi$ and applying the arguments similar to those used in deriving [27, Lemma 2.4], one obtains that there exists $C>0$ independent of $h, \Omega, \gamma$ and $\left\{a_{l_{i}}\right\}_{i=1}^{\Lambda}$ such that

$$
\begin{aligned}
& \left\|\left(\sum_{j \in \mathbb{Z}}\left(\sum_{k \in \mathbb{Z}}\left|\sigma_{h, \Omega, k, \theta, s} * g_{k, j}\right|^{2}\right)^{q / 2}\right)^{1 / q}\right\|_{L^{p}\left(\mathbb{R}^{n}\right)} \\
& \leq C \gamma^{\prime}\|h\|_{\Delta_{\gamma}\left(\mathbb{R}_{+}\right)}\left\|\left(\sum_{j \in \mathbb{Z}}\left(\sum_{k \in \mathbb{Z}}\left|g_{k, j}\right|^{2}\right)^{q / 2}\right)^{1 / q}\right\|_{L^{p}\left(\mathbb{R}^{n)}\right.}
\end{aligned}
$$

for all $1 \leq s \leq \Lambda$ and $(1 / p, 1 / q) \in \mathcal{R}_{\gamma}$. Then (3.53) follows from (3.2), (3.54), (3.56), (3.57), and Lemmas 3.2 and 3.5.

Proof of Theorem 1.2 Let $h, \Omega, P, \varphi$ be given as in Theorem 1.2. By Lemma 2.1, there exist a sequence of complex numbers $\left\{c_{j}\right\}_{j=1}^{\infty}$ and a sequence of $(1, \infty)$ atoms $\left\{\Omega_{j}\right\}_{j=1}^{\infty}$ such that $\Omega=\sum_{j=1}^{\infty} c_{j} \Omega_{j}$ and $\|\Omega\|_{H^{1}\left(S^{n-1}\right)} \approx \sum_{j=1}^{\infty}\left|c_{j}\right|$. In view of the definition of $T_{h, \Omega, P, \varphi}^{*}$,

$$
T_{h, \Omega, P, \varphi}^{*} f \leq \sum_{j=1}^{\infty}\left|c_{j}\right| T_{h, \Omega_{j}, P, \varphi}^{*} f .
$$

In view of (3.58), to prove Theorem 1.2, it suffices to show that there exists $C>0$ independent of $h, \gamma, \Omega$ and the coefficients of $P$ such that

$$
\left\|T_{h, \Omega, P, \varphi}^{*} f\right\|_{L^{p}\left(\mathbb{R}^{n)}\right.} \leq C \gamma^{\prime}\|h\|_{\Delta_{\gamma}\left(\mathbb{R}_{+}\right)}\|f\|_{L^{p}\left(\mathbb{R}^{n}\right)}
$$

holds for any $(1, \infty)$ atom $\Omega$ and $p \in\left(\gamma^{\prime}, \infty\right)$ if $\gamma \geq 2$ or $p \in\left(\gamma^{\prime}, \frac{2 \gamma^{\prime}}{\gamma^{\prime}-2}\right)$ if $\gamma \in(4 / 3,2)$. Let $\Omega$ be a $(1, \infty)$ atom satisfying (2.6)-(2.8) with $0<\varrho \leq 1$ and $\vartheta \in \mathrm{S}^{n-1}$. Without loss of generality, we may assume that $\vartheta=\theta=(1,0, \ldots, 0)$. Let $\left\{\sigma_{h, \Omega, k, \theta, s}\right\}_{s=0}^{\Lambda}$ be given as in the proof of Theorem 1.1. By a simple argument following from the proof of [18, Theorem 2], one has

$$
T_{h, \Omega, P, \phi}^{*} f \leq \sup _{k \in \mathbb{Z}}|| \sigma_{h, \Omega, k, \theta, \Lambda}|* f|+\sup _{k \in \mathbb{Z}}\left|\sum_{j=k}^{\infty} \sigma_{h, \Omega, j, \theta, \Lambda} * f\right| .
$$


By (3.2), (3.54), (3.56), (3.60), and Lemmas 3.2-3.4, we have (3.59) for $p \in\left(\gamma^{\prime}, \infty\right)$ if $\gamma \geq 2$ or $p \in\left(\gamma^{\prime}, \frac{2 \gamma^{\prime}}{\gamma^{\prime}-2}\right)$ if $\gamma \in(4 / 3,2)$.

Proof of Theorem 1.3 Let $h, \Omega, P, \varphi$ be given as in Theorem 1.3. Notice that

$$
\begin{aligned}
\left|\triangle_{\zeta}\left(T_{h, \Omega, P, \varphi}^{*} f\right)(x)\right| & =\left|T_{h, \Omega, P, \varphi}^{*} f(x+\zeta)-T_{h, \Omega, P, \varphi}^{*} f(x)\right| \\
& =\left|T_{h, \Omega, P, \varphi}^{*} f_{\zeta}(x)-T_{h, \Omega, P, \varphi}^{*} f(x)\right| \leq T_{h, \Omega, P, \varphi}^{*}\left(\triangle_{\zeta}(f)\right)(x), \quad \forall x, \zeta \in \mathbb{R}^{n} .
\end{aligned}
$$

This together with Lemma 3.6 and (3.52) implies that, for $\alpha \in(0,1)$ and $1<p, q<\infty$,

$$
\begin{aligned}
& \left\|T_{h, \Omega, P, \varphi}^{*} f\right\|_{\dot{F}_{\alpha}^{p, q}\left(\mathbb{R}^{n}\right)} \\
& \leq C\left\|\left(\sum_{l \in \mathbb{Z}} 2^{l q \alpha}\left(\int_{\mathfrak{R}_{n}}\left|\triangle_{2^{-l} \zeta}\left(T_{h, \Omega, P, \varphi}^{*} f\right)\right| d \zeta\right)^{q}\right)^{1 / q}\right\|_{L^{p}\left(\mathbb{R}^{n}\right)} \\
& \leq C\left\|\left(\sum_{l \in \mathbb{Z}} 2^{l q \alpha}\left(\int_{\mathfrak{R}_{n}}\left|T_{h, \Omega, P, \varphi}^{*}\left(\triangle_{2^{-l} \zeta}(f)\right)\right| d \zeta\right)^{q}\right)^{1 / q}\right\|_{L^{p}\left(\mathbb{R}^{n}\right)} \\
& \leq C \sum_{j=1}^{\infty}\left|c_{j}\right|\left\|\left(\sum_{l \in \mathbb{Z}} 2^{l q \alpha}\left(\int_{\mathfrak{R}_{n}}\left|T_{h, \Omega_{j}, P, \varphi}^{*}\left(\triangle_{2^{-l} \zeta}(f)\right)\right| d \zeta\right)^{q}\right)^{1 / q}\right\|_{L^{p}\left(\mathbb{R}^{n}\right)} .
\end{aligned}
$$

Therefore, to establish the bounds for $T_{h, \Omega, P, \varphi}^{*}$ on $\dot{F}_{\alpha}^{p, q}\left(\mathbb{R}^{n}\right)$, it suffices to show that

$$
\left\|\left(\sum_{l \in \mathbb{Z}} 2^{l q \alpha}\left(\int_{\mathfrak{R}_{n}}\left|T_{h, \Omega, P, \varphi}^{*}\left(\triangle_{2^{-l} \zeta}(f)\right)\right| d \zeta\right)^{q}\right)^{1 / q}\right\|_{L^{p}\left(\mathbb{R}^{n}\right)} \leq C\|f\|_{\dot{F}_{\alpha}^{p, q}\left(\mathbb{R}^{n}\right)}
$$

holds for any $(1, \infty)$ atom $\Omega$ and $\alpha \in(0,1)$ and $1<p, q<\infty$. Here, $C>0$ is independent of $\Omega$ and the coefficients of $P$.

In what follows, let $\Omega$ be a $(1, \infty)$ atom satisfying (2.6)-(2.8) with $0<\varrho \leq 1$ and $\vartheta \in \mathrm{S}^{n-1}$. Without loss of generality, we may assume that $\vartheta=\theta=(1,0, \ldots, 0)$. Let $P,\left\{P_{s}\right\}_{s=0}^{\Lambda},\left\{\Gamma_{s, \theta}\right\}_{s=0}^{\Lambda}$, $\left\{L_{s}\right\}_{s=1}^{\Lambda}$, and $\left\{\sigma_{h, \Omega, k, \theta, s}\right\}_{s=0}^{\Lambda}$ be given as in the proof of Theorem 1.1. We define the measures $\left\{v_{k, s}\right\}_{0}^{2 \Lambda}$ and $\left\{\left|v_{k, s}\right|\right\}_{0}^{2 \Lambda}$ by

$$
\begin{aligned}
& \widehat{v_{k, s}}(\xi)=\int_{2^{k}<|y| \leq 2^{k+1}} \exp \left(\sum_{i=1}^{s} a_{l_{i}} \varphi(|y|)^{l_{i}} \xi \cdot \theta\right) \frac{\Omega(y /|y|)}{|y|^{n}} d y, \quad 0 \leq s \leq \Lambda, \\
& v_{k, s}(\xi)=\sigma_{h, \Omega, k, \theta, s-\Lambda}, \quad \Lambda+1 \leq s \leq 2 \Lambda, \\
& \widehat{\left|v_{k, s}\right|}(\xi)=\int_{2^{k}<|y| \leq 2^{k+1}} \exp \left(\sum_{i=1}^{s} a_{l_{i}} \varphi(|y|)^{l_{i}} \xi \cdot \theta\right) \frac{|\Omega(y /|y|)|}{|y|^{n}} d y, \quad 0 \leq s \leq \Lambda, \\
& \left|v_{k, s}\right|(\xi)=\left|\sigma_{h, \Omega, k, \theta, s-\Lambda}\right|, \quad \Lambda+1 \leq s \leq 2 \Lambda .
\end{aligned}
$$

Let $\xi=\left(\xi_{1}, \ldots, \xi_{n}\right)$. By (1.1) and a change of variable, one has

$$
\widehat{v_{k, s}}(\xi)=0, \quad \forall 0 \leq s \leq \Lambda
$$


Invoking Lemma 2.5, one finds

$$
\left|\widehat{v_{k, s}}\right|(\xi)|=| \int_{2^{k}}^{2^{(k+1)}} \exp \left(\sum_{i=1}^{s} a_{l_{i}} \varphi(t)^{l_{i}} \xi_{1}\right) \frac{d t}{t} \mid\|\Omega\|_{L^{1}\left(S^{n-1}\right)} \leq C\left(\varphi\left(2^{k+1}\right)^{l_{s}}\left|a_{l_{s}} \xi_{1}\right|\right)^{-1 / l_{s}}
$$

Combining this with the trivial estimate ||$\widehat{v_{k, s}}|(\xi)| \leq C$ yields that

$$
\left|\widehat{v_{k, s}}\right|(\xi) \mid \leq C \min \left\{1,\left(\varphi\left(2^{k}\right)^{l_{s}}\left|a_{l_{s}} \xi_{1}\right|\right)^{-1 / l_{s}}\right\}, \quad 1 \leq s \leq \Lambda
$$

By the definition of $\left|v_{k, s}\right|$ and the arguments similar to those used to derive (3.12),

$$
\begin{aligned}
\left|\widehat{v_{k, s}}\right|(\xi) \mid & =|| \widehat{\sigma_{h, \Omega, k, s-\Lambda}}|(\xi)| \\
& \leq C \min \left\{1,\left(\varphi\left(2^{k}\right)^{l_{s-\Lambda}}\left|L_{s-\Lambda}(\xi)\right|\right)^{-1 /\left(2(s-\Lambda) l_{s-\Lambda} \delta\right)}\right\}, \quad \text { for } \Lambda+1 \leq s \leq 2 \Lambda .
\end{aligned}
$$

We get from (3.12) that

$$
\begin{aligned}
\left|\widehat{v_{k, s}}(\xi)\right| & =\left|\widehat{\sigma_{h, \Omega, k, s-\Lambda}}(\xi)\right| \\
& \leq C \min \left\{1,\left(\varphi\left(2^{k}\right)^{l_{s-\Lambda}}\left|L_{s-\Lambda}(\xi)\right|\right)^{-1 /\left(2(s-\Lambda) l_{s-\Lambda} \delta\right)}\right\}, \quad \text { for } \Lambda+1 \leq s \leq 2 \Lambda .
\end{aligned}
$$

One can easily check that

$$
\begin{aligned}
& \left|\widehat{\left|v_{k, s}\right|}(\xi)-\widehat{\left|v_{k, s-1}\right|}(\xi)\right| \\
& \quad=\left|\int_{2^{k}}^{2^{(k+1)}}\left(\exp \left(\sum_{i=1}^{s} a_{l_{i}} \varphi(t)^{l_{i}} \xi_{1}\right)-\exp \left(\sum_{i=1}^{s-1} a_{l_{i}} \varphi(t)^{l_{i}} \xi_{1}\right)\right) \frac{d t}{t}\right|\|\Omega\|_{L^{1}\left(S^{n-1}\right)} \\
& \quad \leq C \varphi\left(2^{k+1}\right)^{l_{s}}\left|a_{l_{s}} \xi_{1}\right| \leq C \varphi\left(2^{k}\right)^{l_{s}}\left|a_{l_{s}} \xi_{1}\right|, \quad \text { for } 1 \leq s \leq \Lambda .
\end{aligned}
$$

Arguments similar to (3.56) show that

$$
\begin{aligned}
\left|\widehat{v_{k, s}}\right|(\xi)-\widehat{\left|v_{k, s-1}\right|}(\xi) \mid & =|| \widehat{\sigma_{h, \Omega, k, \theta, s-\Lambda}}|(\xi)-| \widehat{\sigma_{h, \Omega, k, \theta, s-\Lambda-1}}|(\xi)| \\
& \leq C \min \left\{1, \varphi\left(2^{k}\right)^{l_{s-\Lambda}}\left|L_{s-\Lambda}(\xi)\right|\right\} \quad \text { for } \Lambda+1 \leq s \leq 2 \Lambda .
\end{aligned}
$$

In view of (3.56),

$$
\begin{aligned}
\left|\widehat{v_{k, s}}(\xi)-\widehat{v_{k, s-1}}(\xi)\right| & =\left|\widehat{\sigma_{h, \Omega, k, \theta, s-\Lambda}}(\xi)-\sigma_{h, \widehat{\Omega, k, \theta, s-\Lambda}-1}(\xi)\right| \\
& \leq C \min \left\{1, \varphi\left(2^{k}\right)^{l_{s-\Lambda}}\left|L_{s-\Lambda}(\xi)\right|\right\} \quad \text { for } \Lambda+1 \leq s \leq 2 \Lambda .
\end{aligned}
$$

We now define linear transformations $I_{s}: \mathbb{R}^{n} \rightarrow \mathbb{R}^{n}$ for $1 \leq s \leq 2 \Lambda$ by

$$
I_{s}(\xi)= \begin{cases}a_{l_{s}} \xi_{1} & \text { if } 1 \leq s \leq \Lambda \\ L_{s-\Lambda}(\xi) & \text { if } \Lambda+1 \leq s \leq 2 \Lambda\end{cases}
$$


We also set

$$
\gamma_{s}= \begin{cases}l_{s} & \text { if } 1 \leq s \leq \Lambda \\ l_{s-\Lambda} & \text { if } \Lambda+1 \leq s \leq 2 \Lambda\end{cases}
$$

and

$$
\beta_{s}= \begin{cases}\frac{1}{s l_{s}} & \text { if } 1 \leq s \leq \Lambda \\ \frac{1}{2(s-\Lambda) l_{s-\Lambda} \delta} & \text { if } \Lambda+1 \leq s \leq 2 \Lambda .\end{cases}
$$

It follows from (3.63)-(3.69) that

$$
\begin{aligned}
& \max \left\{\left|\widehat{v_{k, s}}(\xi)-\widehat{v_{k, s-1}}(\xi)\right|,\left|\widehat{v_{k, s}}\right|(\xi)-\widehat{\left|v_{k, s-1}\right|}(\xi) \mid\right\} \leq C\left|2^{k \gamma_{s}} I_{s}(\xi)\right|, \quad 1 \leq s \leq 2 \Lambda \\
& \max \left\{\left|\widehat{v_{k, s}}(\xi)\right|,\left|\widehat{v_{k, s} \mid}(\xi)\right|\right\} \leq C A \min \left\{1,\left|2^{k \gamma_{s}} I_{s}(\xi)\right|^{-\beta_{s}}\right\}, \quad 1 \leq s \leq 2 \Lambda .
\end{aligned}
$$

It is not difficult to see that

$$
\sup _{k \in \mathbb{Z}}|| v_{k, 0}|* f(x)| \leq C|f|(x)
$$

From (3.60) we see that

$$
T_{h, \Omega, P, \varphi}^{*} f \leq \sup _{k \in \mathbb{Z}}|| v_{k, 2 \Lambda}|* f|+\sup _{k \in \mathbb{Z}}\left|\sum_{i=k}^{\infty} v_{i, 2 \Lambda} * f\right| .
$$

Using Lemmas 2.4 and 2.5 in [31], we obtain that, for any $1 \leq s \leq 2 \Lambda$ and $1<p, q, r<\infty$,

$$
\begin{aligned}
& \left\|\left(\sum_{i \in \mathbb{Z}}\left(\sum_{k \in \mathbb{Z}}\left|v_{k, s} * g_{k, i}\right|^{2}\right)^{q / 2}\right)^{1 / q}\right\|_{L^{p}\left(\mathbb{R}^{n}\right)} \leq C\left\|\left(\sum_{i \in \mathbb{Z}}\left(\sum_{k \in \mathbb{Z}}\left|g_{k, i}\right|^{2}\right)^{q / 2}\right)^{1 / q}\right\|_{L^{p}\left(\mathbb{R}^{n}\right)} ; \\
& \left\|\left(\sum_{i \in \mathbb{Z}}\left\|\left(\sum_{k \in \mathbb{Z}}|| v_{k, s}\left|* g_{i, \zeta, k}\right|^{2}\right)^{1 / 2}\right\|_{L^{r}\left(\Re_{n}\right)}^{q}\right)^{1 / q}\right\|_{L^{p}\left(\mathbb{R}^{n}\right)} \\
& \quad \leq C\left\|\left(\sum_{i \in \mathbb{Z}}\left\|\left(\sum_{k \in \mathbb{Z}}\left|g_{i, \zeta, k}\right|^{2}\right)^{1 / 2}\right\|_{L^{r}\left(\Re_{n}\right)}^{q}\right)^{1 / q}\right\|_{L^{p}\left(\mathbb{R}^{n}\right)} .
\end{aligned}
$$

By (3.63), (3.70)-(3.72), (3.74), (3.75) and invoking Lemma 3.7, we have that, for $\alpha \in$ $(0,1), 1<p, q<\infty$, and $1 \leq s \leq 2 \Lambda$,

$$
\begin{aligned}
& \left\|\left(\sum_{l \in \mathbb{Z}} 2^{l q \alpha}\left(\int_{\mathfrak{\Re}_{n}} \sup _{k \in \mathbb{Z}}|| v_{k, s}|*| \triangle_{2^{-} l_{\zeta}} f|| d \zeta\right)^{q}\right)^{1 / q}\right\|_{L^{p}\left(\mathbb{R}^{n)}\right.} \leq C\|f\|_{\dot{F}_{\alpha}^{p, q}\left(\mathbb{R}^{n}\right)}, \\
& \left\|\left(\sum_{l \in \mathbb{Z}} 2^{l q \alpha}\left(\int_{\mathfrak{\Re}_{n}} \sup _{k \in \mathbb{Z}}\left|\sum_{i=k}^{\infty} v_{i, 2 \Lambda} * \triangle_{2^{-} l_{\zeta}} f\right| d \zeta\right)^{q}\right)^{1 / q}\right\|_{L^{p}\left(\mathbb{R}^{n}\right)} \leq C\|f\|_{\dot{F}_{\alpha}^{p, q}\left(\mathbb{R}^{n}\right)} .
\end{aligned}
$$

Then (3.62) follows from (3.73), (3.76), and (3.77). Furthermore, the boundedness for $T_{h, \Omega, P, \varphi}^{*}$ on $F_{\alpha}^{p, q}\left(\mathbb{R}^{n}\right)$ follows from the boundedness for $T_{h, \Omega, P, \varphi}^{*}$ on $\dot{F}_{\alpha}^{p, q}\left(\mathbb{R}^{n}\right),(2.4),(2.5)$, and Theorem 1.2. By (3.61), (3.62) and the arguments similar to those used in deriving the 
continuity part of [31, Theorem 1.1], we can get the continuity part in Theorem 1.3. This completes the proof of Theorem 1.3.

\author{
Acknowledgements \\ The authors want to express their sincere thanks to the referee for his or her valuable remarks and suggestions, which \\ made this paper more readable.
}

\title{
Funding
}

The first author was supported by Young Teachers and Top-Notch Talents in Undergraduate Teaching of Shandong University of Science and Technology (No. BJRC20180502). This second author was supported partially by the NNSF of China (No. 11701333)

\section{Availability of data and materials}

Not applicable.

\section{Declarations}

\section{Competing interests}

The authors declare that they have no competing interests.

\section{Authors' contributions}

YLZ was a major contributor in writing the manuscript. FL performed the validation and formal analysis. All authors read and approved the final manuscript.

\section{Publisher's Note}

Springer Nature remains neutral with regard to jurisdictional claims in published maps and institutional affiliations.

Received: 27 October 2021 Accepted: 13 January 2022 Published online: 28 January 2022

\section{References}

1. Al-Hasan, A., Pan, Y.: LP-boundedness of a singular integral operator. Can. Math. Bull. 41(4), 404-412 (1998)

2. Al-Qassem, A., Cheng, L., Pan, Y.: Boundedness of rough integral operators on Triebel-Lizorkin spaces. Publ. Math. 56, 261-277 (2012)

3. Al-Salman, A., Pan, Y.: Singular integrals with rough kernels in $L \log L\left(\mathrm{~S}^{n-1}\right)$. J. Lond. Math. Soc. 66(2), 153-174 (2002)

4. Calderón, A.P., Zygmund, A.: On singular integral. Am. J. Math. 78, 289-309 (1956)

5. Chen, J., Fan, D., Ying, Y.: Singular integral operators on function spaces. J. Math. Anal. Appl. 276, 691-708 (2002)

6. Chen, J., Zhang, C.: Boundedness of rough singular integral on the Triebel-Lizorkin spaces. J. Math. Anal. Appl. 337 , 1048-1052 (2008)

7. Chen, P., Duong, X.T., Li, J., Wu, Q.: Compactness of Riesz transform commutator on stratified Lie groups. J. Funct. Anal. 277, 1639-1676 (2019)

8. Chen, W., Fu, Z., Grafakos, L., Wu, Y.: Fractional Fourier transforms on $L^{p}$ and applications. Appl. Comput. Harmon. Anal. 55, 71-96 (2021)

9. Chen, Y., Ding, Y.: Rough singular integrals on Triebel-Lizorkin space and Besov space. J. Math. Anal. Appl. 347, 493-501 (2008)

10. Chen, Y., Ding, Y., Liu, H.: Rough singular integrals supported on submanifolds. J. Math. Anal. Appl. 368, 677-691 (2010)

11. Cheng, L.C.: Singular integrals related to homogeneous mappings. Mich. Math. J. 47, 407-416 (2000)

12. Coifman, R.R., Weiss, G.: Extensions of Hardy spaces and their use in analysis. Bull. Am. Math. Soc. 83(6), $569-645$ (1977)

13. Colzani, L.: Hardy spaces on sphere. PhD Thesis, Washington University, St Louis, MO (1982)

14. Colzani, L., Taibleson, M., Weiss, G.: Maximal estimates for Cesàro and Riesz means on sphere. Indiana Univ. Math. J. 33(6), 873-889 (1984)

15. Connett, W.C.: Singular integrals near $L^{1}$ in harmonic analysis in Euclidean spaces. In: Proc. Sympos. Pure Math., pp. 163-165. Am. Math. Soc., Providence (1979)

16. Duoandikoetxea, J., Rubio de Francia, J.L.: Maximal and singular integral operators via Fourier transform estimates. Invent. Math. 84(3), 541-561 (1986)

17. Fan, D., Guo, K., Pan, Y.: Singular integrals with rough kernels along real-analytic submanifolds in $\mathbb{R}^{n}$. Integral Equ. Oper. Theory 33, 8-19 (1999)

18. Fan, D., Guo, K., Pan, Y.: A note of a rough singular integral operator. Math. Inequal. Appl. 2(1), $73-81$ (1999)

19. Fan, D., Guo, K., Pan, Y.: Singular integrals with rough kernels along real-analytic submanifolds in $\mathbb{R}^{3}$. Trans. Am. Math. Soc. 355(3), 1145-1165 (2003)

20. Fan, D., Pan, Y.: L²-boundedness of a singular integral operator. Publ. Mat. 41, 317-333 (1997)

21. Fan, D., Pan, Y.: A singular integral operator with rough kernel. Proc. Am. Math. Soc. 125, 3695-3703 (1997)

22. Fan, D., Pan, Y.: Singular integral operators with rough kernels supported by subvarieties. Am. J. Math. 119(4), 799-839 (1997)

23. Fefferman, R.: A note on singular integrals. Proc. Am. Math. Soc. 74(2), 266-270 (1979)

24. Frazier, M., Jawerth, B., Weiss, G.: Littlewood-Paley Theory and the Study of Function Spaces. CBMS Reg. Conf. Ser., vol. 79. Am. Math. Soc., Providence (1991)

25. Grafakos, L., Stefanov, A.: $L^{p}$ bounds for singular integrals and maximal singular integrals with rough kernels. Indiana Univ. Math. J. 47, 455-469 (1998) 
26. Liu, F.: On singular integrals associated to surfaces. Tohoku Math. J. 66(1), 1-14 (2014)

27. Liu, F.: Rough singular integrals associated to surfaces of revolution on Triebel-Lizorkin spaces. Rocky Mt. J. Math. 47(5), 1617-1653 (2017)

28. Liu, F., Mao, S., Wu, H.: On rough singular integrals related to homogeneous mappings. Collect. Math. 67(1), 113-132 (2016)

29. Liu, F., Wu, H.: Singular integrals related to homogeneous mappings in Triebel-Lizorkin spaces. J. Math. Inequal. 11(4), $1075-1097$ (2017)

30. Liu, F., Xue, Q., Yabuta, K.: Rough maximal singular integral and maximal operators supported by subvarieties on Triebel-Lizorkin spaces. Nonlinear Anal. 171, 41-72 (2018)

31. Liu, F., Xue, Q., Yabuta, K.: Boundedness and continuity of maximal singular integrals and maximal functions on Triebel-Lizorkin spaces. Sci. China Math. 63(5), 907-936 (2020)

32. Namazi, J.: A singular integral. Proc. Am. Math. Soc. 96, 201-219 (1986)

33. Ricci, F., Stein, E.: Harmonic analysis on nilpotent groups and singular integrals I: oscillatory integrals. J. Funct. Anal. 73 179-194 (1987)

34. Sato, S.: Estimates for singular integrals and extrapolation. Stud. Math. 192, 219-233 (2009)

35. Shi, S., Fu, Z., Lu, S.: On the compactness of commutators of Hardy operators. Pac. J. Math. 307, $239-256$ (2020)

36. Shi, S., Xiao, J.: Fractional capacities relative to bounded open Lipschitz sets complemented. Calc. Var. Partial Differ. Equ. 56, 1-22 (2017)

37. Stein, E.M.: Problems in harmonic analysis related to curvature and oscillatory integrals. In: Proceedings of the International Congress of Mathematicians, pp. 196-221. Amer. Math. Soc., Providence (1987)

38. Stein, E.M.: Harmonic Analysis: Real-Variable Methods, Orthogonality, and Oscillatory Integrals. Princeton University Press, Princeton (1993)

39. Stein, E.M.: Some geometrical concepts arising in harmonic analysis. Geom. Funct. Anal. Special volume, Part 1, 434-453 (2000)

40. Triebel, H.: Theory of Function Spaces. Monogr. Math., vol. 78. Birkhäser, Basel (1983)

41. Yabuta, K.: Triebel-Lizorkin space boundedness of Marcinkiewicz integrals associated to surfaces. Appl. Math. J. Chin. Univ. 30(4), 418-446 (2015)

42. Yang, M., Fu, Z., Sun, J.: Existence and large time behavior to coupled chemotaxis fluid equations in Besov-Morrey spaces. J. Differ. Equ. 266, 5867-5894 (2019)

43. Zhang, C., Chen, J.: Boundedness of singular integrals and maximal singular integrals on Triebel-Lizorkin spaces. Int. J. Math. 21(2), 157-168 (2010)

\section{Submit your manuscript to a SpringerOpen ${ }^{\circ}$ journal and benefit from:}

- Convenient online submission

- Rigorous peer review

- Open access: articles freely available online

- High visibility within the field

- Retaining the copyright to your article

Submit your next manuscript at $\boldsymbol{s p r i n g e r o p e n . c o m ~}$ 